\title{
Effects of Sampling on Stability and Performance of Electronically Controlled Pressure Reducing Valves
}

\author{
Tomasz Janus ${ }^{1}$ and Bogumil Ulanicki ${ }^{2}$ \\ ${ }^{1}$ Department of Mechanical, Aerospace and Civil Engineering, The University of Manchester, \\ George Begg Bldg, Manchester, M1 3BB, UK (corresponding author), Email: \\ tomasz.janus@manchester.ac.uk \\ ${ }^{2}$ Water Software Systems, School of Engineering and Sustainable Development, De Montfort \\ University, The Gateway, LE1 9BH, Leicester, UK
}

\section{ABSTRACT}

This paper explains and demonstrates how increasing a sampling period in pressure control may worsen system's performance and may lead to instability. Notion of stability of continuous-time and discrete-time systems is briefly introduced and applied to a simple closed-loop inertial system. It is then demonstrated how stability of dynamic systems depends on a sampling period as well as on gain. Subsequently, the analysis is applied to a model of an electronically controlled pressure reducing valve (PRV) coupled with a transient model of a water distribution network (WDN). Occurrence of instabilities at overly long sampling periods is demonstrated. Practical recommendations on the appropriate choice of sampling times are given based on the simulation results and control engineering rules of thumb given the closed-loop system's dynamics. Performance of a theoretical pressure control scheme is then simulated under time-varying demands and with controllers designed to work at different sampling frequencies.

\section{INTRODUCTION}

Pressure control is an important task in management of WDNs and has two main functions: (1) servo control which tracks time-varying pressure setpoint in a given node of a network in 
order to, e.g. minimize leakage, and (2) regulation control which maintains the pressure setpoint irrespective of disturbances, e.g. sudden demand changes in the network or events such as pump or valve switching, hydrant opening or bursts. These two functions impose somewhat different requirements on the dynamics of the pressure control system and shall be discussed in detail in this manuscript.

So far, most of the publications on real-time control (RTC) in WDNs predominantly considered pressure management for leakage control and demand supply whilst disturbance rejection was covered only in a handful of papers, e.g. (Creaco et al. 2017; Creaco et al. 2018). Excessive pressure in a WDN leads to elevated levels of pressure-dependent background leakage (Vicente et al. 2016) and increases the risk of bursts (Thornton and Lambert 2010). On the other hand, insufficient pressure in parts of the network may result in a reduction or a complete loss of service to some consumers. Pressure control in WDNs is most often accomplished via PRVs which are usually installed at the inlets to district metering areas (DMAs) and are designed to maintain the desired outlet pressure. The pressure setpoint can either be constant or follow a time trajectory, which can either be scheduled or calculated on-line in an outer control loop as in e.g. Fontana et al. (2018). As water demands in the network follow a diurnal pattern, pressures in the network will vary accordingly. Background leakage depends on slowly varying pressure trends in the network and hence, the speed of a servo controller can be relaxed. Consequently, the times between consecutive control actions can be in a range of minutes, provided that the controller is designed appropriately, as shall be discussed in this manuscript. For constant inlet pressure, the pressures further in the network will be higher during low-demand periods and lower during high-demand periods. This may be undesirable if the intention is to keep the pressures within a DMA at quasi-constant nearoptimal levels. One solution to this problem is to modulate the PRV setpoint as a function of flow, which was done by AbdelMeguid et al. (2011) using a special hydraulic device installed in a bypass circuit of a hydraulically operated PRV. Another solution is to manipulate the PRVs based on pressure measurements from within the network using electronic controllers. This was proposed in a number of publications - see Creaco et al. (2019) for a detailed review of the work in this field. In 
case of hydraulic PRVs the manipulated variable is the pressure setpoint adjusted by the pilot valve screw which fulfills the servo control function, whilst the inner hydraulic circuit via pilot valve performs regulatory function, i.e. disturbance rejection.

Recently, some water companies have been introducing pressure control schemes where valve elements are actuated directly in feedback control loops with electronic controllers combined with electro-mechanical actuators. We shall call these valves remotely controlled valves (RCVs). RCVs belong to the class of PRVs. In such valves, which are usually installed in strategic supply points in a WDN, both servo control and regulatory control are implemented using electronic control circuits. For disturbance rejection, contrary to servo control, regulators need to react to inputs of high frequencies resulting from e.g. sudden changes in demands, e.e. hydrant openings or valve and pump switching, which in turn necessitates short time-steps between consecutive control actions. These disturbances need to be rejected, because even though they may be short-lived and thus, have only a small impact on background leakage, they can be detrimental to water distribution network infrastructure and water quality. Pressure transients put strain on pipes and may cause new bursts (Malppan and Sumam 2015; Rezaei et al. 2015). They may also lead to worsened water quality by dislodging materials deposited on the pipe walls, e.g. biofilms (S.Jones et al. 2015). In this solution, an appropriate choice of sampling times for both control loops becomes a valid engineering problem which the authors aim to answer in this manuscript. Moreover, the issue of appropriate selection of sampling times in digital control systems is not only restricted to PRVs but is valid also for other applications including pump speed control.

The literature on RTC of WDNs is abundant and has recently been reviewed and summarized by Creaco et al. (2019). The bulk of the publications on the subject are often based on static network models (Campisano et al. 2010; Campisano et al. 2012; Creaco and Franchini 2013; Campisano et al. 2016; Giustolisi et al. 2017; Page et al. 2017b; Page et al. 2017a; Creaco 2017; Page et al. 2018; Berardi et al. 2018; Page and Creaco 2019). The authors investigated reduction of leakage using different control strategies and controllers within an extended period simulation (EPS) environment where the implemented control algorithms would adjust the valve setting in between successive 
steady-state solutions. Since the focus was placed on water savings resulting from RTC and not on pressure control at shorter time-scales characteristic of the disturbances, the authors of the above papers used long control time steps which necessitated using small gains (i.e. slow control action) in order to maintain stability, as shall be explained further in this manuscript. Specifically, time steps of $3 \mathrm{~min}$ in e.g. Campisano et al. (2012) and $5 \mathrm{~min}$ in e.g. Giustolisi et al. (2017) were the most frequently chosen values. Dynamic behavior of the network was not considered and it was instead assumed that the chosen control time step would be sufficiently long such that the transients resulting from the valve adjustments would fade away between successive control actions. Whilst the papers cited above provide insight into potential water savings resulting from RTC, removal of time dimension from the hydraulic models and thus, of inertial (rigid-column) and wave (water hammer) dynamics did not allow the authors to investigate how the proposed control schemes would behave under time-varying demands at shorter time-scales. Although the controller was claimed to be proportional it is in fact a discrete integral controller since its form $\Delta a_{i}=K_{p} \Delta h_{i}$ implies that the control signal $a=K_{p} \sum_{i} \Delta h_{i}$, i.e. is proportional to the sum of the errors over the previous timesteps not the current value of the error, as in proportional controllers. As shall be discussed further, the choice of sampling times in a range of minutes required the use of rather small gain values in order to reduce the bandwidth and maintain appropriate stability margins. The proposed control schemes were designed for the purpose of responding to low frequency pressure signal components for leakage management. They are unable to respond to higher frequency signals resulting from demand changes, which require controllers with larger bandwidths, i.e. higher gain values and correspondingly, shorter sampling periods. As shall be demonstrated, stability of discrete-time closed-loop systems depends on the dynamics of the open-loop system (without feedback) and, for a given system and a given controller, is a function of the open-loop static gain (denoted as $K$ ) as well as the sampling period $T$.

Recently, some of the authors discussed implementations of the aforementioned control algorithms using transient WDN simulation models and analyzed their performances at different control time steps. Using a form of a valve model-derived control algorithm from Creaco and Franchini 
(2013), Creaco et al. (2017) reported a stable control system at time steps $\Delta t=180$ s and longer but oscillatory behavior at $\Delta t=3 \mathrm{~s}$. The authors concluded that $\Delta t=180 \mathrm{~s}$ was a good choice for a control interval. However, the proposed control system was tested only during the time period where demand was relatively constant and thus its disturbance rejection properties could not be assessed. Creaco et al. (2018), using control time steps $\Delta t=1 \mathrm{~min}$ and $\Delta t=3 \mathrm{~min}$ in combination with a transient hydraulic model, noted an improvement with a smaller time step out of the two but then concluded that if the control time was overly reduced, control instabilities could arise. The authors tested the dynamic response of their remotely-controlled valve to activation of a hydrant but, due to a long control time step and small gain warranting stability at that particular case, the system was unable to maintain adequate pressure levels and pressure variations up to $10 \mathrm{~m}$ were observed. Similar conclusions were reiterated in Page and Creaco (2019) who reported instability at $\Delta t \leq 1 \mathrm{~min}$ and found optimal behavior of their system at $\Delta t=3 \mathrm{~min}$. Galuppini et al. (2019) used a sampling time of $1 \mathrm{~s}$ but then concluded that longer sampling intervals might be preferable. In contrast to the above, other publications using transient models, e.g. Prescott and Ulanicki (2003, Prescott and Ulanicki (2008, AbdelMeguid et al. (2011, Madoński et al. (2014, Janus and Ulanicki (2018) or based on experimental studies (AbdelMeguid et al. 2011; Fontana et al. 2018) quoted much smaller sampling periods in the range of milliseconds.

The goal of this paper is to highlight the importance of proper selection of sampling times in feedback control systems in general and specifically, when applied to pressure control in WDNs, and to describe the mechanisms by which sampling time can be properly selected and the impact of sampling on stability properly assessed. Initially, basic definition of stability are provided and the reader is introduced to the concept of local stability analysis at equilibrium points via model linearization. Conditions for stability of the linearized linear time invariant (LTI) systems in continuous-time and discrete-time together are given. After introducing the necessary theory it is demonstrated how a simple first order LTI system which is inherently stable, becomes unstable after adding a feedback loop in which the error signal is sampled with a too large a sampling period. It is also shown that that it is possible to have a stable closed-loop system with very long 
sampling periods, provided that the static gain is sufficiently low. However, such a system would also become slower and with lesser ability to follow reference signals and reject disturbances. Later in the paper, the results of this simple simulation study will become more evident when more theory is introduced and the readers are familiarized with Bode plots and the concepts of bandwidth and stability margins. The main part of this paper is focused on the dynamics of a model of a closedloop pressure control system with an electronically controlled PRV installed in a full-size WDN described with a transient hydraulic model and solved with a method of characteristics. In order to design an electronic controller and check its closed-loop stability at different sampling times the transient model was identified for a number of operating points, producing a family of LTI models represented by transfer functions. As shall be shown later, the identified LTI models allow us to formally describe the WDN dynamics. Operation of the designed controllers at different sampling periods was then tested on the transient simulation model using step inputs in the reference signal as well as under time-varying demands.

The work presented here is motivated by a real-life incident which happened in a pressure control scheme in a major UK city and which was the subject of a paper by Janus and Ulanicki (2017). Pressure at the inlet of the WDN was controlled by a valve whose position was adjusted by an electromechanical actuator combined in a feedback loop with a proportional integral (PI) controller. It was observed that the gain in the feedback loop increased significantly under low valve openings causing instability in the system under low-flow conditions. At the same time it was also observed that stability was sensitive to the chosen sampling time.

Nominal and robust stability of pressure control systems was recently discussed in detail in Galuppini et al. (2020). The study was focused on continuous-time systems, albeit the effects of sampling were also explained, while this work deals specifically with discrete-time systems. The analysis was carried out on reduced models of first order plus delay although the authors later stressed the need for higher order models. This work considers a higher order model with additional reduction of static nonlinearity via gain compensation. Galuppini et al. (2020) considered a control structure in which the valve position is directly controlled on a pressure reading from a sensor 
located farther in the network and used a Smith predictor for delay compensation. In practice, it is known that Smith compensator is very sensitive to time delay errors to the point that, for given robustness levels, standard proportional integral derivative (PID) controllers usually outperform Smith predictors even for simple first-order processes with delay (Sigurd Skogestad 2018). In WDNs time delay could vary as a result of changes to network topology, e.g. states of isolation valves in the network and addition/removal of pipes, gradual changes to pipe properties, and possibly also as a result of variability in distribution of demands across the network. In Galuppini et al. (2020) the reported performance from the Smith predictor however, was satisfactory.

This work advocates controlling the valve based on the pressure signal measurement taken in the proximity of the valve due to destabilizing effects of delay, as described in this paper. Since perfect delay compensation is difficult to achieve, positioning the pressure sensor away from the controlled element introduces unwanted delay uncertainty and thus reduces robustness. Ultimately, the controller needs to have a smaller closed-loop bandwidth to ensure robust stability compared to the scenario where the sensor was positioned closer to the valve. Instead, one could use a cascaded control structure in which the valve is controlled based on the pressure reading taken close to the valve whilst the setpoint is controlled based on the pressure reading deeper in the network. This configuration allows separation of controller dynamics, such that the inner loop controller can be made faster for disturbance rejection (regulation) whilst reference tracking (servo) loop can be designed to track slowly varying pressures for leakage management, ultimately resulting in a faster and more robust control system.

\section{CONTROL SYSTEM STRUCTURE}

The closed-loop pressure control system used in this study is best described with a block diagram shown in Fig. 1 where arrows represent signals and blocks represent dynamic elements. By convention, signals are functions in time domain $t$ while system dynamics are represented in the complex frequency domains $s$ and $z$ as transfer functions. A brief introduction to transfer functions, Laplace and $\mathcal{Z}$ transforms are provided further down in the text. Fig. 1 shows two alternative open-loop paths formed by (a) analog controller $C(s)$, actuator $A(s)$ and plant $P(s)$ and 
(b) $P(s), C(s)$ and $A(s)$ preceded by a sampler followed by a zero-order hold (ZOH) element with transfer function $G_{h}(s)$. The measured pressure head $h_{d}(t)$ downstream of the PRV is fed back to the summation node and compared with the desired reference downstream pressure head $h_{r e f}(t)$. The resulting error signal $e(t)=h_{r e f}(t)-h_{d}(t)$ forms the input to the controller which produces the output $u(t)$ driving the actuator that sets the valve position $x_{m}(t)$. In case of (b) the error signal is sampled at discrete-time intervals with a sampling period $T$ producing a series of pulses $e(k T)$ where $k$ is an integer. The $\mathrm{ZOH}$ block holds the signal constant between each pulse resulting in a stair-shaped error signal $\bar{e}(t)$. During sampling and subsequent $\mathrm{ZOH}$ reconstruction some of the information about the error signal $e(t)$ is irrevocably lost. Instead of the actual error value the controller receives the information outdated by up to one sample. What follows is that the actuator sets the valve position $\bar{x}_{m}(t)$ based on the outdated (between the samples) control signal $\bar{u}(t)$. If the sampling time $T$ is small enough, the difference between the true and the sampled error signal will be negligible and the dynamics of the continuous-time and the discrete-time systems will be similar. As $T$ becomes larger and the controller acts on more outdated information the quality of control in the sampled system will deteriorate. It is easy to guess that the system is more likely to become unstable if (a) the controller receives more outdated information about the output, i.e. $T$ grows larger and (b) the controller reacts stronger upon each computation of the control signal, i.e. the controller is faster.

\section{STABILITY OF DYNAMICAL SYSTEMS}

If a dynamic system is stable then the system outputs are bounded within admissible limits under bounded inputs, the so-called bounded input bounded output (BIBO) stability. The converse of the above statement however may not always be true for the reasons beyond the scope of this paper and related to the presence of the so-called transmission zeros. In water systems, instability of a feedback loop may manifest itself through the valve remaining fully open or fully closed (saturation) or through sustained periodic oscillations. Sometimes, a more strict description would be that the system states tend to an equilibrium point of interest (the so-called asymptotic stability). Asymptotic stability of a system implies BIBO stability introduced above. Theory of stability 
features different problem-specific definitions of stability. In our discussion of a feedback control system we shall use the notion of stability of a system with respect to its equilibrium points. We shall restrict our discussion to continuous-time and discrete-time single input single output (SISO) systems since a valve/WDN can be regarded as a SISO system with the valve position $x_{m}$ being the input and the downstream head at the valve outlet $h_{d}$ being the output. In continuous-time a SISO control system can be described with the following state-space equations

$$
\begin{aligned}
& \dot{\mathbf{x}}(t)=\mathbf{f}(\mathbf{x}(t), u(t)) \\
& y(t)=\mathbf{g}(\mathbf{x}(t), u(t))
\end{aligned}
$$

where $\mathbf{x}(t) \in \mathbf{R}^{n}$ is the state vector of the system, $y(t) \in \mathbf{R}$ is the (scalar) output of the system, $u(t) \in \mathbf{R}$ is the (scalar) control input, $\mathbf{f}$ is a continuously differentiable nonlinear function and $\mathbf{g}$ is a continuous or smooth nonlinear function. In discrete-time, a nonlinear SISO control system is described with a discrete-time state-space model of the form

$$
\begin{gathered}
\mathbf{x}_{k+1}=\mathbf{f}\left(\mathbf{x}_{k}, u_{k}\right) \\
y_{k}=\mathbf{g}\left(\mathbf{x}_{k}, u_{k}\right)
\end{gathered}
$$

where all notations are similarly defined and $k=0,1, \ldots$ denote the sample numbers.

An equilibrium point of a system with control input $u$ is a solution to the algebraic set of equations $\mathbf{f}\left(\mathbf{x}^{*}, u^{*}\right)=0$ in case of continuous-time systems and $\mathbf{x}^{*}=\mathbf{f}\left(\mathbf{x}^{*}, u^{*}\right)$ in case of discretetime systems and is given as a pair $\left(\mathbf{x}^{*}, u^{*}\right)$. In other words, an equilibrium point is a state of the system in which the derivatives are zero. By definition, $\dot{\mathbf{x}}^{*}=0$ and $\dot{u}^{*}=0$. In many cases, the above sets of equations are undetermined as different constant control inputs $u^{*}$ (scalar in case of a SISO system considered here) will produce different equilibriums $\mathbf{x}^{*}$ in state-space. For the purpose of introducing stability definitions, which are usually given in literature for systems without control 
inputs, let us remove $u^{*}$ from $\left(\mathbf{x}^{*}, u^{*}\right)$ and refer to $\mathbf{x}^{*}$ as the system's equilibrium point corresponding to some constant given input $u^{*}$. Additionally, let us shift the origin of the coordinate system in state-space such that non-zero equilibrium point is located at the origin and consider $\mathbf{x}^{*}=0$ as the system's equilibrium point corresponding to a constant input $u^{*}$.

Then the following definitions of a local stability in the vicinity of an equilibrium point can be formulated. For the continuous-time system described by Eq. 1:

Definition 3.1 (Stability in continuous-time) The system $\dot{\mathbf{x}}(t)=\mathbf{f}(\mathbf{x}(t), u(t))$ is stable in the sense of Lyapunov about its equilibrium $\mathbf{x}^{*}=0$ under constant input $u^{*}$ if for any $\varepsilon>0$ and any initial time $t_{0}$ there exists a ball $\delta\left(\varepsilon, t_{0}\right)>0$, such that $\left\|\mathbf{x}\left(t_{0}\right)\right\|<\delta \Rightarrow\|\mathbf{x}(t)\|<\varepsilon$ for all $t \geq t_{0}$.

Definition 3.2 (Asymptotic stability in continuous-time) The system $\dot{\mathbf{x}}(t)=\mathbf{f}(\mathbf{x}(t), u(t))$ is asymptotically stable about its equilibrium $\mathbf{x}^{*}=0$ under constant input $u^{*}$ if it is stable in the sense of Lyapunov and, furthermore, there exists a constant $\delta=\delta\left(t_{0}\right)>0$, such that $\left\|\mathbf{x}\left(t_{0}\right)\right\|<\delta \Rightarrow\|\mathbf{x}(t)\| \rightarrow 0$ as $t \rightarrow \infty$.

Analogously, for the discrete-time system in Eq. 2:

Definition 3.3 (Stability in discrete-time) The system $\mathbf{x}_{k+1}=\mathbf{f}\left(\mathbf{x}_{k}, u_{k}\right)$ is stable in the sense of Lyapunov about its equilibrium $\mathbf{x}^{*}=0$ under a constant input $u^{*}$ if for any $\varepsilon>0$ and any initial $k_{0}$ there exists a ball $\delta\left(\varepsilon, k_{0}\right)>0$, such that $\left\|\mathbf{x}_{k_{0}}\right\|<\delta \Rightarrow\left\|\mathbf{x}_{k}\right\|<\varepsilon$ for all $k \geq k_{0}$.

Definition 3.4 (Asymptotic stability in discrete-time) The system $\mathbf{x}_{k+1}=\mathbf{f}\left(\mathbf{x}_{k}, u_{k}\right)$ is asymptotically stable about its equilibrium $\mathbf{x}^{*}=0$ under a constant input $u^{*}$ if it is stable in the sense of Lyapunov and, furthermore, there exists a constant $\delta=\delta\left(k_{0}\right)>0$, such that $\left\|\mathbf{x}_{k_{0}}\right\|<\delta \Rightarrow\left\|\mathbf{x}_{k}\right\| \rightarrow 0$ as $k \rightarrow \infty$.

where $t_{0}$ is the initial time, $k_{0}$ is the initial index, and $\|\cdot\|$ denotes the Euclidean norm. Stability in the sense of Lyapunov means, in simple terms, that the solution which starts near an equilibrium point $\mathbf{x}^{*}$ will stay near $\mathbf{x}^{*}$ forever. Asymptotic stability additionally implies that the solution not only stays near $\mathbf{x}^{*}$ but also converges to it. 
Let us now consider the systems in Eq. 1 and Eq. 2, respectively, linearized around the equilibrium point $\left(\mathbf{x}^{*}, u^{*}\right)$.

$$
\begin{gathered}
\dot{\bar{x}}(t)=\mathbf{A} \overline{\mathbf{x}}(t)+\mathbf{B} \bar{u}(t) \\
\bar{y}(t)=\mathbf{C} \overline{\mathbf{x}}(t)+\mathbf{D} \bar{u}(t) \\
\overline{\mathbf{x}}_{k+1}=\mathbf{A} \overline{\mathbf{x}}_{k}+\mathbf{B} \bar{u}_{k} \\
\bar{y}_{k}=\mathbf{C} \overline{\mathbf{x}}_{k}+\mathbf{D} \bar{u}_{k}
\end{gathered}
$$

where the system matrix $\mathbf{A}=[\partial \mathbf{f} / \partial \mathbf{x}]_{\left(\mathbf{x}^{*}, \mathbf{u}^{*}\right)}$, the input matrix $\mathbf{B}=[\partial \mathbf{f} / \partial \mathbf{u}]_{\left(\mathbf{x}^{*}, \mathbf{u}^{*}\right)}$, the output matrix $\mathbf{C}=[\partial \mathbf{g} / \partial \mathbf{x}]_{\left(\mathbf{x}^{*}, \mathbf{u}^{*}\right)}$, and the feedthrough matrix $\mathbf{D}=[\partial \mathbf{g} / \partial \mathbf{u}]_{\left(\mathbf{x}^{*}, \mathbf{u}^{*}\right)}$ and $\overline{\mathbf{x}}(t)=\mathbf{x}(t)-\mathbf{x}^{*}$, $\bar{u}(t)=u(t)-u^{*}, \bar{y}(t)=y(t)-y^{*}, \overline{\mathbf{x}}_{k}=\mathbf{x}_{k}-\mathbf{x}^{*}, \bar{u}_{k}=u_{k}-u^{*}, \bar{y}_{k}=y_{k}-y^{*}$ are the deviations of the state, input, and output in continuous-time and discrete-time, respectively.

According to the first Lyapunov criterion, stability analysis of an equilibrium point $\left(\mathbf{x}^{*}, u^{*}\right)$ can be carried out by studying the stability of the corresponding linearized system in the vicinity of $\left(\mathbf{x}^{*}, u^{*}\right)$. The criterion applies to continuous-time as well as discrete-time systems.

Theorem 3.1 (First Method of Lyapunov) Consider the two nonlinear dynamic systems in statespace forms defined in Eq. 1 (continuous-time) and Eq. 2 (discrete-time) and their linearized forms in Eq. 3 and Eq. 4, respectively, determined around the equilibrium point $\left(\mathbf{x}^{*}, u^{*}\right)$. For the two considered systems, the following statements hold: If all the eigenvalues of $\mathbf{A}$ have negative real parts (in continuous-time) / modulus less than one (in discrete-time), then the corresponding nonlinear system is asymptotically stable about $\left(\mathbf{x}^{*}, u^{*}\right)$. On the other hand, if $\mathbf{A}$ has at least one eigenvalue with a positive real part (in continuous-time) / with a modulus greater than one (in discrete-time) then the corresponding nonlinear system is unstable around $\left(\mathbf{x}^{*}, u^{*}\right)$. 
In other words, it is enough to check if a linearized version of a nonlinear system around an equilibrium point is asymptotically stable in order to ensure (local) convergence to that equilibrium point. On the other hand, if the linearized system is unstable then the original nonlinear system will also be unstable around that equilibrium point. Theorem 3.1 is inconclusive when $\mathbf{A}$ has eigenvalues on the imaginary axis (in continuous-time) or with modulus equal one (in discrete-time) - i.e. for border-line situations - see Strogatz (2007). In these cases, higher order terms neglected during linearization around the equilibrium point determine whether this equilibrium point is stable or unstable.

We can think of the above theorems in the context of a simple pendulum. In the vertically inverted upside-down position with the center of mass above its pivot point at an angle of $180^{\circ}$ the pendulum is in an unstable equilibrium because the slightest perturbation that deviates the angle from $180^{\circ}$ will cause the pendulum to fall over and eventually reach a (new) stable equilibrium with the center of mass below its pivot point at an angle $0^{\circ}$, i.e. with the pendulum facing down. The equilibrium is asymptotically stable because it is reached in finite time - see Definition 3.2 and Definition 3.4. We may think of a scenario in which the pendulum is in an idealized environment with no air resistance and no friction in the pivot point. If the starting point is with the pendulum in its stable equilibrium, i.e. with the pendulum facing down and its position is deviated by some angle $\phi \mid<180^{\circ}$ then the pendulum will remain oscillating around the stable equilibrium within bounds $[-|\phi|,+|\phi|]$. The equilibrium is stable because the deviation does not grow in time but no longer asymptotically stable as it will not be reached in finite time - see Definition 3.1 and Definition 3.3.

\section{Linear Time Invariant systems and frequency domain}

Linear (or linearized) time invariant systems can be conveniently transformed (under certain conditions beyond the scope of this paper) from time domain $t$ to complex frequency domain $s=\sigma+j \omega$ and from discrete-time domain $k T$ to complex frequency domain $z \stackrel{\text { def }}{=} e^{s T}=e^{(\sigma+j \omega) T}$. The transformations $y(t) \stackrel{\mathcal{L}}{\mapsto} Y(s)$ and $y[k] \stackrel{\mathcal{Z}}{\mapsto} Y(z)$ are carried out using $\mathcal{L}$ and $\mathcal{Z}$ transforms, respectively (Ogata 2010). State-space systems in Eq. 3 and Eq. 4 in time domain become 
$Y(s) / U(s)=\mathbf{C}(s \mathbf{I}-\mathbf{A})^{-1} \mathbf{B}+\mathbf{D}$ and $Y(z) / U(z)=\mathbf{C}(z \mathbf{I}-\mathbf{A})^{-1} \mathbf{B}+\mathbf{D}$ in frequency domain, respectively. The produced algebraic output-input ratios $Y(s) / U(s)=\sum_{k=0}^{M} b_{k} s^{k} / \sum_{k=0}^{N} a_{k} s^{k}$ and $Y(z) / U(z)=\sum_{k=0}^{M} b_{k} z^{k} / \sum_{k=0}^{N} a_{k} z^{k}$ are ratios of polynomials in $s$ and $z$ and are called transfer functions $G(s)$ and $H(z)$, respectively. Denominators $D(s)=\sum_{k=0}^{N} a_{k} s^{k}$ and $D(z)=\sum_{k=0}^{N} a_{k} z^{k}$ of $G(s)$ and $H(z)$ describe the homogeneous parts of their corresponding ordinary differential equation (ODE) and difference equation (DE) and determine their stability. It can be shown, e.g. Ogata (2010) that roots of $D(s)$ and $D(z)$, from now on called the poles $p_{i}$ of $G(s)$ and $H(z)$ are equal to the eigenvalues $\lambda_{i}$ of $\mathbf{A}$ when the system has no transmission zeros, i.e. when the state space system is a minimal representation of the transfer function. This is the case for the system studied here and for this reason, from now on stability will be considered in frequency domain. Thus, stability conditions for transfer functions are analogous to those for LTI state-space systems see the First Method of Lyapunov in Theorem 3.1. In continuous-time, the transfer function model is stable iff all its poles $p_{i}$ satisfy the condition $\mathfrak{R}\left(p_{i}\right)=\sigma_{i}<0$. In discrete-time, the transfer function is stable iff all its poles $p_{i}$ satisfy the condition $\left|p_{i}\right|<1$. In both cases, the stability is asymptotic.

Whilst pole locations give us information about absolute stability or lack thereof of a linear or linearized system, relative stability provides information about the proximity of a system to instability after closing the feedback loop and can be calculated or measured in frequency $s$ or $z$ domains. Two stability margins are defined. The gain margin (GM) is the amount of gain that can be added in the open-loop system before the closed-loop system (with added feedback) becomes unstable. The phase margin (PM) is the amount of extra phase lag (phase shift) that is allowed in the open-loop system before the closed-loop system becomes unstable. Both margins can be determined and visualized on Nyquist or Bode plots and formal GM and PM definitions can be found in e.g. Ogata (2010). In this paper, we shall use Bode plots which show the system's gain vs frequency and the system's phase vs frequency on two separate subplots. The destabilizing effects of gain on pressure control systems was already discussed in Janus and Ulanicki (2018). In this paper, we shall discuss the destabilizing effects of phase lag, which can be introduced in 
the system as delays and/or via sampling. For this purpose, a closed-loop pressure control system is designed for a theoretical WDN. The controller design procedure is as follows: First, the nonlinear valve/WDN system is linearized around the desired equilibrium. Next, linear controllers are designed for the derived linear model at different sampling times. In accordance with 3.1 if the linear model is closed-loop stable, so will be the nonlinear system in the neighborhood of the equilibrium. Ultimately, the designed closed-loop control systems are checked in a simulation to see if the controllers meet the design criteria on the nonlinear model. In order to prevent the integral of the error increasing in case the input to the plant saturates due to e.g. nonlinearities such as rate limiters, the controller with integral action needs to be equipped with integrator anti-windup logic.

\section{Stability analysis of a simple first order sampled system}

Let us quickly visualize how feedback in closed-loop feedback control systems may destabilize an otherwise stable open-loop system when the sampling period is too long and/or the static gain is too large. For this purpose we will use the simplest dynamic system, i.e. first-order inertia described with a differential equation $\tau \frac{d}{d t} y(t)+y(t)=K u(t)$ and whose transfer function $G(s)=Y(s) / U(s)=K /(\tau s+1)$ where $K$ is the static gain and $\tau$ is the time constant. The closedloop system shown in Fig. 2 can be viewed as an analogy to a feedback pressure control scheme where $G(s)$ represents the inertial response of a rigid-column hydraulic model (Janus and Ulanicki 2017). The system is preceded with a $\mathrm{ZOH}$ block with transfer function $G_{h}(s)=\left(1-e^{-T s}\right) / s$ and an ideal sampler with sampling period $T$. The open-loop transfer function (without feedback) after the sampler can be written as $G_{\text {open }}(s)=G_{h}(s) G(s)=K\left(1-e^{-T s}\right) /(s(\tau s+1))$. The open-loop transfer function of the sampled system (including the sampler) can be calculated by taking an inverse $\mathcal{L}$ transform of $G_{\text {open }}(s)$ and then converting the resulting difference equation into the corresponding $\mathcal{Z}$ transfer function, $H_{\text {open }}(z)=\mathcal{Z}\left\{\mathcal{L}^{-1}\left[G_{\text {open }}(s)\right]_{t=k T}\right\}$. After carrying out the appropriate calculations - see e.g. Vande Vegte (1986), the following closed-loop transfer function of the sampled system is obtained

$$
H_{\text {closed }}(z)=K\left(1-e^{-T / \tau}\right) /\left[z-e^{-T / \tau}+K\left(1-e^{-T / \tau}\right)\right]
$$


Since the system is $1^{\text {st }}$ order it has a single pole $p$ and is stable if $|p|<1$. After equating the denominator $D(z)$ to zero $|p|=\left|e^{-T / \tau}-K\left(1-e^{-T / \tau}\right)\right|<1$. Solving the inequality produces the following stability criterion for our sampled $1^{\text {st }}$ order system: $T<\tau \ln [(K+1) /(K-1)]$. The maximum allowed sampling time depends on the system's time constant $\tau$ and the open-loop static gain $K$. The time constant $\tau$ is directly linked to bandwidth in frequency domain, which shall be discussed later. The bigger the $\tau$, i.e. the smaller the closed-loop bandwidth, the larger $T$ can get before the system becomes unstable. The relationship versus $K$ is the opposite since $K$ makes the closed-loop response faster, not slower, as in case of $\tau$. Let us assume the system with $K=2$ and $\tau=0.4 \mathrm{~s}$. The above condition gives $T \lesssim T_{\max }=0.44 \mathrm{~s}$.

The system's response $y(t)$ to a unit step in the reference signal $r(t)=1$ was simulated for a sampling period $T=4 \mathrm{~s}$ sufficiently long to reach new steady-state outputs in between samples and for $T=0.4$ s slightly smaller than the calculated (stable) maximum value. The results plotted in Fig. 3 show that for $T=4 \mathrm{~s}$, as expected, the system is unstable and the amplitude of oscillation increases with every sample. In this case, sampling is too infrequent to correct the course of the system and the absolute value of the error $|e|=|r-y|$ increases with each sample. When $T$ is reduced to a value slightly below $T_{\max }$ the system becomes stable, albeit with frequent oscillations as $T$ is still very close to $T_{\max }$ - see Fig. $3 \mathrm{~b}$. The response will become less oscillatory when $T$ is further reduced until it finally resembles an exponential curve. The derived stability criterion can be reordered to find the maximum value of $K$ for a given $T: K<\left(e^{T / \tau}+1\right) /\left(e^{T / \tau}-1\right)$. The system can theoretically be stable for large $T$ 's provided that $K$ is sufficiently small. This explains how some of the publications quoted in the Introduction produced stable solutions at very long time-steps.

The results in Fig. 3 can be explained without any of the above mathematical formalism by looking at the output $\left(y_{k}\right)$ and the error $\left(e_{k}\right)$ values at consecutive samples. We shall take the advantage of the fact that the static gain of $1 /(\tau s+1)$ is equal one and $T=4 \mathrm{~s}$ is sufficiently long for the system to attain new steady-state in between the samples. Hence, in the $k$-th sample the error $e_{k}=r_{k}-x_{k-1}$ whilst the new steady-state output $y_{k}=K e_{k}$. At the start of the iteration $r_{0}=0$, 
$e_{0}=0$, and $y_{0}=0$. For $k \geq 1, r_{k}=1$ representing a unit-step in the input. At $K=2$ and sampling time $T=4$ s (see Fig. $3 \mathrm{a}$ and the first time series in Table 1) the steady-state output value at each sample $y_{k}$ is always twice the current error $e_{k}$ which leads to gradual amplification of the error signal and the output itself. The system is unstable. It is intuitive that the system will be stable if the gain $K<1$ such that the initial error $e_{k}$ at sample $k=1$ is gradually attenuated instead of amplified. At $K=1$ the system is marginally stable and the error and the output signals are oscillating between zero and one (see the second time series in Table 1). To turn an unstable closed-loop system into a stable one the open-loop gain needs to be reduced so that the error signal $e_{k}$ is attenuated instead of amplified or, alternatively, one can reduce the sampling time such that the system response does not have time to grow between consecutive control actions. The step response of the system in Fig. 2 at $T=0.4$ s can be conveniently calculated whilst taking advantage of the fact that $T=\tau$ where, by definition, time constant $\tau$ is the time required for the system to reach $(1-1 / e) \approx 63.2 \%$ of the new steady-state value. Therefore, in the $k$-th sample, $e_{k}=r_{k}-y_{k-1}$ whilst the output at the end of the sample $y_{k}=y_{k-1}-\left(y_{k-1}-K e_{k}\right)(1-1 / e)$. At the end of each sample the output attains only $63.2 \%$ of its final value and the error signal $e_{k}=r_{k}-x_{k-1}$ becomes smaller than it would have been if the sampling time was longer. Consequently, the system becomes stable as shown in Fig. $3 \mathrm{~b}$ and the third time series in Table 1.

\section{IDENTIFICATION OF THE PRV/WDN SYSTEM}

The closed-loop pressure control system was designed for a WDN consisting of a skeletonized network model described with waterhammer equations, a static hydraulic model of a PRV and an inertial model describing the dynamics of the actuator. The combined PRV/WDN model was simulated to generate the input-output data used to identify a family of continuous-time transfer functions between the input to the actuator $u(t)$ and the system output $y(t)=h_{d}(t)$, i.e. the measured downstream pressure head. Dynamic responses to a unit step in $u(t)$ were computed at different operating points defined by the combination of demands and the downstream pressure $h_{d}=h_{\text {ref }}$ in closed-loop. Consequently, a family of transfer functions were identified, covering all tested operating points. 


\section{Description of the network simulation model}

As shown in the schematic diagram in Fig. 4, the network model is composed of 42 pipes and 28 nodes. The pipes between nodes 4 and 28 are each of $75 \mathrm{~mm}$ diameter and $200 \mathrm{~m}$ length whilst the two pipes upstream and downstream of the PRV are, respectively, 4000m and 400m in length and both $200 \mathrm{~mm}$ in diameter. It is assumed that pressure waves propagate with a constant velocity $c=1200 \mathrm{~m} / \mathrm{s}$ uniform across the network. Friction is modeled with Darcy-Weisbach formula with friction factor $f=2 \times 10^{-4}$ assumed to remain constant within the range of Reynolds numbers observed in the network. The nodes are assigned different elevations ranging between $z_{i}=100 \mathrm{~m}$ in node 3 to $z_{i} \in[10,50] \mathrm{m}$ in nodes 4 to 28 .

Demand $d_{i}$ at each node $i$ is a sum of pressure-independent demand $d_{i}^{p i}$ and pressure-dependent demand $d_{i}^{p d}$. It can be argued that all demands in a transient model should be pressure-dependent since forcing demands independently of pressure does not reflects the physics of fluid flow, which is pressure driven (Janus and Ulanicki 2017). Doing so leads to overestimation of the magnitude of pressure waves (Jung et al. 2009) and, as shown later in Fig. 5, also the shape of the dynamic response. Although efforts have been made to propose a mathematical description of pressuredependent demands (Giustolisi and Walski 2012) the application has so far been limited to steadystate WDN analysis. Pressure-dependency of demands in non-steady state conditions is much more elaborate and since our modeling efforts are only aimed at demonstrating the concept of closed-loop system stability in the practical context of pressure control in WDNs, not on exact description of a particular network, the proposed approach seems adequate. For the same reason, pressure-dependent demand in each node $i$ is calculated with the power equation $d_{i}^{p d}=C_{i}\left(h_{i}-z_{i}\right)^{\alpha}$ where $C_{i}$ is the pressure-dependent demand coefficient in node $i$ and $\alpha=1$ is the network-wide pressure-dependent demand exponent, instead of the more accurate FAVAD formula (van Zyl and Cassa 2014). In the unsteady simulation case study, $d_{i}^{p i}$ were generated using the bottom up stochastic approach by Buchberger and Wells (1996).

Pressure loss in the PRV $\Delta h_{P R V}=q_{m}^{2} / C_{v}\left(x_{m}\right)^{2}$ where $q_{m}\left(\mathrm{~m}^{3} / \mathrm{s}\right)$ denotes the flow across the valve and $C_{v}\left(x_{m}\right)\left(\mathrm{m}^{2.5} / \mathrm{s}\right)$ is the valve coefficient which depends on valve opening $x_{m}(\%)$. The 
valve coefficient $C_{v}\left(x_{m}\right)=0.02107-0.02962 e^{-0.0140 x_{m}}+0.0109 e^{-0.0713 x_{m}}-0.00325 e^{-0.1866 x_{m}}+$ $0.0009 e^{-0.1091 x_{m}}$ is calculated using a regression curve derived from the manufacturer's data. The actuator is described with a first order transfer function $A(s)=1 /\left(\tau_{A} s+1\right)$ where the actuator's time constant $\tau_{A}=0.6 \mathrm{~s}$.

\section{Identification procedure and results}

Model identification was carried out in MATLAB $^{\circledR}$ using the System Identification Toolbox $^{\mathrm{TM}}$ function tfest which, for continuous-time transfer functions, estimated on time-domain data, uses State-Variable Filters (SVFs) combined with the simplified refined Instrument Variable (IV) method for estimable parameter initialization (Ljung 2009). The transfer function models between the input to the actuator $u$ and the downstream pressure head $h_{d}$ were identified, one per each operating point, for a total number of 18 operating points defined by: pressure-dependent demand coefficient $C_{i} \in[2,4,8] \times 10^{-6} \mathrm{~m}^{2} / \mathrm{s}$, pressure-independent demand $d_{i}^{p i} \in[0.014,0.026,0.036] \mathrm{m}^{3} / \mathrm{s}$ and the outlet pressure head setpoint $h_{\text {ref }} \in[140,155] \mathrm{m}$. For simplicity, it was assumed that $C_{i}$ and $d_{i}^{p i}$ are equal in all nodes at a given operating point. The resulting combinations of $d_{i}^{p i}$ and $C_{i}$ produced $q_{p d} \in\left[0.08 q_{t o t}, 0.55 q_{t o t}\right]$, i.e. the pressure-dependent demand constituted between $8 \%$ and $55 \%$ of the total flow in the network.

After attaining a steady-state for a selected combination of $C_{i}, d_{i}^{p d}$ and $h_{r e f}$ in closed-loop, the feedback loop was removed and a unit step signal $u(t)=1$ was fed to the actuator in open-loop. Whilst the choice of excitation (input) signal for identification is not trivial and there are many options (Ljung 1999) the unit step signal was chosen for its simplicity and ease of interpretation of results in time domain. The measured output of the transient model, i.e. the pressure head $h_{d}$ downstream of the PRV was recorded for each step input. The recorded input-output data was fed to tfest in order to estimate the coefficients $a_{i}$ and $b_{i}$ of the continuous transfer function of the form shown in Eq. 6.

$$
G(s) A(s)=\frac{b_{2} s^{2}+b_{1} s+b_{0}}{a_{4} s^{4}+a_{3} s^{3}+a_{2} s^{2}+a_{1} s+a_{0}}
$$

$G(s) A(s)$ is a $4^{\text {th }}$ order transfer function with 2 zeros and 4 poles and with one 'slow' pole 
originating from the actuator dynamics. It can be argued that since our transient hydraulic model is infinite-dimensional and with complex wave dynamics, its approximation is likely to be of relatively high order. However, pressure control systems are usually set up with actuators and opening rate limiters having slow enough dynamics to prevent induction of faster (higher order) pressure wave oscillations in the network. These elements act as low-pass filters attenuating higher order dynamics and producing an overall lower-order dynamic response. It is also hypothesized that higher order dynamics which can be observed in individual pipes or small networks are lesser in larger and more complex networks due to the pressure waves being attenuated at individual nodes as the waves meeting each other are likely to have different phases. Since the actuator used in our study has a bandwidth $\omega_{A}=1.66 \mathrm{rad} \approx 0.26 \mathrm{~Hz}$ with a $20 \mathrm{~dB} /$ decade magnitude roll-off afterwards any transient frequencies above $1.5 \mathrm{~Hz}$ will be scaled down to less than $17 \%$ of their original amplitudes. The significance of the system having two zeros is that the dynamic response of $G(s) A(s)$ does not only depend on the velocity of the valve opening but also on its acceleration.

Six out of 18 recorded step responses (bold) with the corresponding outputs from the identified transfer functions (thin) are plotted in Fig. 5. The responses have different static gains which are dependent on the valve position $x_{m, 0}$ at the start of the step experiment, but rather similar dynamics, i.e. rise times, amounts of overshoot, settling times and peak times. Some difference in dynamic response can be noticed in step responses obtained at higher $C$ values, i.e. with higher pressuredependent demand component (see Fig. 5b). These responses seem to have a slightly different shape to those obtained for lower $C$ values, plotted in Fig. 5a, and show distinct step-like departure and arrival moments of the principal pressure wave.

In order to get a better understanding of the dynamics of the identified transfer functions, their frequency characteristics are plotted in a form of a Bode plot in Fig. 6a. The magnitude subplot of the Bode plot shows the differences in amplitudes, i.e. static gains, which predominantly depend on the valve position, while the phase characteristics are closer together indicating similar dynamics across the family of the identified models. The only differences in phase characteristics can be observed around $\sim 0.5-0.7 \mathrm{rad} / \mathrm{s}$. These frequencies are suspected to be responsible for the initial 
part of the time-response and the variability around these frequencies is attributed to the earlier mentioned differences in the dynamic response at different $C$ coefficients. For better visualization, the plots were divided into three separate series plotted with: dashed line for $x_{m} \leq 35 \%$, solid line for $35 \%<x_{m} \leq 50 \%$, and dashed-dotted line for $x_{m}>50 \%$. The Bode plot was generated up to the Nyquist frequency $\omega_{N} \approx 157.1 \mathrm{rad} / \mathrm{s}=\pi / 0.02 \mathrm{~s}$ where $h=0.02 \mathrm{~s}$ is the time step in the fixed-step hydraulic solver employed in the method of characteristics. Again, it can be seen that lower gains coincide with higher valve openings whilst higher gains were recorded under lower valve openings.

\section{CONTROLLER DESIGN IN CONTINUOUS-TIME}

\section{Gain compensator design}

In order to keep the open-loop static gain $K$ of the system approximately constant under all operating conditions the authors implemented the methodology presented in Janus and Ulanicki (2018) and designed a gain compensator block which scales the input to the actuator based on the measurement of the valve position. $K$ values measured during valve operation were were plotted against valve position $x_{m}$ in Fig. 7 together with the fitted regression curve $K\left(x_{m}\right)=p_{1} e^{-p_{2} x_{m}}$, where $p_{1}=29.2, p_{2}=0.0636$ with confidence intervals $\langle 19.9,38.4\rangle$ and $\langle 0.0551,0.0722\rangle$, respectively. The curve shows a good quality of fit with root mean squared error (RMSE) of 0.242 confirming the earlier finding that the static gain of the PRV connected to a WDN is predominantly dependent on the valve position. Each identified transfer function $G(s) A(s)$ can be described as a product of its static gain $K$ and the dynamic part with unity static gain, as shown in Eq. 7 .

$$
G(s) A(s)=K \frac{b_{2}^{*} s^{2}+b_{1}^{*} s+1}{a_{4}^{*} s^{4}+a_{3}^{*} s^{3}+a_{2}^{*} s^{2}+a_{1}^{*} s+1}
$$

where $b_{i}^{*}=b_{i} / b_{0}$ and $a_{i}^{*}=a_{i} / a_{0}$. Each one out of the 18 identified transfer functions has a different $K$ which, when multiplied by the gain compensator $k\left(x_{m}\right)$ defined below, should become close to a typical gain $K_{t y p}$ used for controller tuning. As in Janus and Ulanicki (2018) the gain compensator is calculated as follows: $k\left(x_{m}\right)=K\left(x_{m}=x_{t y p}\right) / K\left(x_{m}\right)$ where $x_{t y p}$ denotes a typical 
(average) valve position (here $x_{t y p}=50 \%$ ) and $K\left(x_{m}\right)$ is our regression curve. The Bode plot of the gain compensated system $k\left(x_{m}\right) G(s) A(s)$ is shown in Fig. 6b. The curves displayed in bold represent the nominal gain compensated plant and actuator transfer function given in Eq. 8 .

$$
k\left(x_{m}\right) G(s) A(s)=1.20 \frac{7.944 s^{2}+0.8332 s+1.703}{s^{4}+7.036 s^{3}+10.06 s^{2}+2.971 s+1.355}
$$

which was selected from the family of the identified transfer functions for valve position $x_{m}=52.9 \%$ for which $k\left(x_{m}\right)=1.20$. Fig. $6 \mathrm{~b}$ demonstrates that the designed gain compensator is effective as the individual magnitude plots now lie much closer together. The steady-state gains of the step responses now fall between 1.0 and 1.8 which means $\sim 6 \times$ smaller spread of values compared to the uncompensated system.

\section{Controller design for the gain compensated system}

A continuous-time integral (I) controller with transfer function $C(s)=K_{i} / s$ was tuned using MATLAB $^{\circledR}$ 's pidtune function on the nominal system given in Eq. 8 for a chosen target 0dB gain crossover frequency of the tuned open-loop response $\omega_{c}=0.15 \mathrm{rad} / \mathrm{s}$. The crossover frequency $\omega_{c}$ approximately sets the control bandwidth and the closed-loop response time $\approx 1 / \omega_{c}$, The tuned I controller gain $K_{i}=0.0985$ leads to a closed-loop system with gain margin $G M=36.6 \mathrm{~dB}$ at $3.03 \mathrm{rad} / \mathrm{s}$ and phase margin $P M=74.2^{\circ}$ at $0.15 \mathrm{rad} / \mathrm{s}$. In time domain (not shown) the resulting nominal closed-loop system response has $0 \%$ overshoot (OS), zero steady-state error, settling time $t_{s}=26 \mathrm{~s}$ and rise time $t_{r}=9.7 \mathrm{~s}$. The definitions of the above terms can be found in one of the control engineering textbooks, e.g. (Ogata 2010; Phillips and Harbor 2000; Vande Vegte 1986). The gain and phase margins are significant which safeguards the system against modeling uncertainties and drift with regards to gain amplification and delay. This indicates a safe and robust controller. The nominal closed-loop transfer function $G_{\text {closed }}(s)=G_{\text {open }}(s) /\left(1+G_{\text {open }}(s)\right)$ where $G_{\text {open }}(s)=1.20 C(s) G(s) A(s)$ is given in Eq. 9 in the zero-pole-gain form, i.e. with 
factored polynomials in the nominator and the denominator.

$$
G_{\text {closed }}(s)=\frac{0.939\left(s^{2}+0.1049 s+0.2144\right)}{(s+5.253)(s+1.415)(s+0.1971)\left(s^{2}+0.1717 s+0.1374\right)}
$$

$G_{\text {closed }}(s)$ is a stable $5^{\text {th }}$ order system with 3 real and 2 complex conjugate poles and 2 complex conjugate zeros. There are two fast poles: one at -5.3 corresponding to a time constant $\tau=0.19 \mathrm{~s}$ and the second one at -1.4 , i.e. with $\tau=0.70$ s, and one slow (dominant) real pole at -0.197 ( $\tau=5.1 \mathrm{~s})$. The two complex conjugate poles producing low-frequency low-amplitude oscillations are located near the two complex conjugate zeros. These two pairs are responsible for a notch and a peak around $0.37 \mathrm{rad} / \mathrm{s}$ and $0.46 \mathrm{rad} / \mathrm{s}$, respectively, in the frequency subplot of the Bode plot in Fig. 6b. The system becomes unstable when the open-loop static gain is magnified 67.6 times, which in $\log$ scale, is equal to $36.6 \mathrm{~dB}$, i.e. the gain margin. The nominal closed-loop system has a large stability margin but nevertheless, as we shall see later, becomes unstable when the output/error signal is sampled with $T>(15-20)$ s, i.e. $1.5-2.0$ times the closed-loop rise time $t_{r}$ which, for the nominal system, is $\sim 9.7$ seconds.

\section{CLOSED-LOOP PERFORMANCE AND STABILITY IN DISCRETE-TIME DOMAIN}

Each $j$-th identified open-loop transfer function in continuous-time $G_{o p e n, j}(s)$ where $G_{o p e n, j}(s)=$ $k\left(x_{m}\right) K_{i} / s[G(s) A(s)]_{j}$ was transformed into an open-loop discrete-time transfer function $H_{o p e n, j}(z)$ at different sampling periods $T$. The corresponding closed-loop transfer function was then calculated using the formula analogous to the one in continuous-time, $H_{\text {closed }}(z)=H_{\text {open }}(z) /\left(1+H_{\text {open }}(z)\right)$. As shown in Fig. 8, the poles of the nominal closed-loop transfer function move away from the origin in the $z$-plane as $T$ increases until one of the poles goes out of the unit circle for $T \approx 16.5 \mathrm{~s}$, thus rendering the closed-loop system unstable.

The closed-loop time responses of the family of 18 identified transfer functions with the tuned I controller to a step change in the reference signal $h_{\text {ref }}$ are plotted in Fig. 9 for different sampling periods. At $T=1 \mathrm{~s}$ the responses have a small $\sim 1 \% \mathrm{OS}$ and settling times of $<40 \mathrm{~s}$. As $T$ increases the responses become more oscillatory with larger settling times and larger overshoots. At $T=15 \mathrm{~s}$ 
the nominal system is still stable, albeit with a large OS of $\sim 100 \%$ and a settling time of $>120$ s. Although the nominal system loses stability at $T \gtrsim 16.5 \mathrm{~s}$ (as demonstrated in Fig. 8), some of the systems identified at different operating points are unstable already at $T=15 \mathrm{~s}$ due to slight differences in dynamics and imperfect gain compensation.

The performance of the tuned I controller combined with the gain compensator at different sampling periods was ultimately tested on the transient WDN model. The results in Fig. 10 show that at $T=1 \mathrm{~s}$ the closed-loop response is similar to the one with the identified transfer function model. It is only slightly more oscillatory due to some neglected fast dynamics in the transfer function approximation. The similarity begins to fall apart at larger sampling periods for which the transient PRV/WDN becomes unstable already at $T=10 \mathrm{~s}$, whilst at $T=5 \mathrm{~s}$ it is already quite oscillatory with OS of up to $70 \%$. Dynamics neglected during the identification of (lower order) transfer functions begin to play a role in the overall discrete-time system stability, however at already long and practically infeasible sampling periods. At $T=0.25 \mathrm{~s}$ the closed-loop performance with the transient WDN model is very similar to the one with the identified transfer function and in continuous-time. This suggests that, for this particular controller, the system should be sampled with $T \approx 0.25-0.30$ s and not less frequently than $T=1-1.5$ s or otherwise, stability margins will be significantly reduced in comparison to their designed values and the overall system response will grow oscillatory up to the point of eventually becoming unstable.

Reduction of closed-loop stability margins at larger sampling periods and static gains are visualized in Bode plots in Fig. 11 using the nominal plant in open loop with the tuned I controller. As previously, the plots are generated up to the Nyquist frequency marked with a vertical solid line. The closed-loop system's phase margin (PM) is the additional amount of phase lag that is required for the open-loop system's phase to reach $-180 \mathrm{deg}$ at the the gain crossover frequency $\omega_{g c}$, i.e. the frequency where the open-loop system's magnitude is $0 \mathrm{~dB}$. Likewise, the gain margin $(\mathrm{GM})$ is the additional amount of gain required for the open-loop system's magnitude to reach $0 \mathrm{~dB}$ at the phase crossover frequency $\omega_{p c}$, i.e. the frequency where the open-loop system's phase equals $-180 \mathrm{deg}$. As we can see in Fig. 11, increasing the sampling time curves the phase plot downwards whilst 
the magnitude plot remains relatively unchanged up to frequencies close to the Nyquist frequency. Consequently, PM is reduced as the phase curve lies closer to -180 deg (from above) and GM is also reduced due to the fact that phase crossover occurs at lower frequencies $\omega_{p c}$ while, for most physical systems, magnitude of a transfer function decreases with frequency. This global trend can be reversed locally since the function may not always be monotonic, as described further below. On the other hand, increasing the static gain shifts the magnitude plot upwards whilst the phase plot remains the same. Consequently, GM decreases as the magnitude plot lies closer to to $0 \mathrm{~dB}$ (from below) while PM is usually reduced as it is evaluated at higher gain crossover frequencies $\omega_{g c}$ and phase decreases with frequency, albeit not always monotonically, as mentioned above with regards to the magnitude plot. In this case study, we can see a notch and a peak for frequencies $\omega \approx 0.3-1.1 \mathrm{rad} / \mathrm{s}$ due to the effects of wave dynamics on top of inertial dynamics. As a result, the phase locally increases for $\omega \approx 0.5-1.1 \mathrm{rad} / \mathrm{s}$. Consequently, PM will not change monotonically with static gain and we can have a larger PM for $K=10$ than for $K=5$. However, we need to bear in mind that we deal with a family of models with different frequency characteristics, depending on the operating point (see Fig. 6b) and with significant differences in phase plots between the models in this particular frequency range. Additionally, we need to safe guard the system against the effects of unmodeled dynamics and thus, for this particular sampling period $T=1 \mathrm{~s}$, we would not want to design a controller with a closed-loop bandwidth $\omega_{c} \approx \omega_{g c} \gtrsim 0.15 \mathrm{rad} / \mathrm{s}$. As static gain is increased, so is the gain crossover frequency $\omega_{g c}$ whilst phase crossover frequency $\omega_{p c}$ remains the same. Since, $\omega_{g c}<\omega_{c}<\omega_{p c}$, but as a rule of thumb $\omega_{c} \approx \omega_{g c}$, as static gain is increased, so is the closed-loop bandwidth $\omega_{c}$ and thus, we have a closed-loop system which is more responsive inputs of higher frequencies.

\section{CASE STUDY}

\section{Controller design at different sampling intervals}

As discussed above, for a given controller with constant gains, there exists a maximum sampling period beyond which the closed-loop system becomes unstable. In order to be able to use larger sampling periods whilst keeping the system robust, i.e. stable and with sufficient gain and phase 
margins, the response of the open-loop system needs to be made slower or, in frequency domain terminology, the target closed-loop bandwidth $\omega_{c}$ needs to be reduced. To find the target $\omega_{c}$ value for a given discrete controller with sampling time $T$ it will be assumed that $\omega_{c}$ is a fraction $f_{\omega_{c}}$ of Nyquist frequency $\frac{\pi}{T}$, i.e. $\omega_{c}=\frac{\pi}{f_{\omega_{c}} T}$. The gain and phase margins of the systems designed with integral (I) controllers at different $T=(1,5,15,60) \mathrm{s}$ and $f_{\omega_{c}}$ are plotted in Fig. 12. As $f_{\omega_{c}}$ increases so do the phase and gain margins of a discrete-time system. To safeguard against static gain increase a target GM of approx. $15-25 \mathrm{~dB}$ requires $f_{\omega_{c}} \approx 25$. For this value of $f_{\omega_{c}} \mathrm{PM}$ is approx. $75^{\circ}-85^{\circ}$. For a given $f_{\omega_{c}}$ phase margins tend to be smaller for the systems designed for higher target $\omega_{c}$ due to the presence of higher order dynamics in the dynamic response. At lower target $\omega_{c}$ values ( $T=15 \mathrm{~s}$ and $T=60 \mathrm{~s}$ ), the valve/WDN response is approximately inertial and the PM curves in Fig. 12 are near each other. In this case study, $f_{\omega_{c}}=25$ and the resulting target $\omega_{c}=\left(1.257 \times 10^{-1}, 2.513 \times 10^{-2}, 8.378 \times 10^{-3}, 2.094 \times 10^{-3}, 6.981 \times 10^{-4}, 4.189 \times 10^{-4}\right) \mathrm{rad} / \mathrm{s}$ for each target $T$, respectively. The resulting I controller gains for each $T$ respectively, are as follows: $K_{i}=\left(8.274 \times 10^{-2}, 1.666 \times 10^{-2}, 5.555 \times 10^{-3}, 1.389 \times 10^{-3}, 4.629 \times 10^{-4}, 2.777 \times\right.$ $\left.10^{-4}\right) \mathrm{m} /(\% \mathrm{~s})$. As can be seen in Fig.6b the chosen target frequencies are below the frequencies of some key system dynamics present at $\omega>0.1 \mathrm{rad} / \mathrm{s}$ which approximate the wave dynamics of the full (transient) system. Thus, it can be expected, and shall be verified via simulation, that the designed closed-loop system will not be able to compensate for some transient effects due to sudden demand changes. Since the target closed-loop system responses are slow, the I controller suffices to satisfy the desired response and addition of proportional (P) and derivative (D) terms of the PID controller did not make any improvements to the overall response of the closed-loop system. In order to compare the performance of the above closed-loop systems against ones with faster loop dynamics, three controllers at $T=(0.01,0.20,0.50) \mathrm{s}$ were also designed. In contrast to the above, the presence of higher order dynamics necessitated using higher order (PID) controllers. The calculated gain triplets $\left(K_{p}, K_{i}, K_{d}\right)$ for the three controllers are $(1.308,2.324,0.1840),(1.018,0.2724,0.2083)$, and $(0.1422,0.1277,0)$, respectively. These controllers (in continuous-time) were chosen to guarantee infinite gain margins (GM) and phase 
margins $\mathrm{PM}=80^{\circ}$ at $\omega_{c}=2 \mathrm{rad} / \mathrm{s}, 0.4 \mathrm{rad} / \mathrm{s}$, and $0.2 \mathrm{rad} / \mathrm{s}$, respectively. It can be seen in Fig. $6 \mathrm{~b}$ that $\omega_{c}=2 \mathrm{rad} / \mathrm{s}$ lies to the right of the notch and peak in the phase plot representing some crucial system dynamics and therefore, it is expected that the system will be better able to reject higher frequency disturbances. The choice of target $\omega_{c}$ values was less automated than in the former case with longer sampling times and necessitated some manual iterations to satisfy the chosen stability margins and response characteristics in presence of more complex (wave) dynamics in the system. The resulting step responses exhibit overshoots $(\mathrm{OS})$ of $<2.5 \%$ for $\mathrm{T}=0.2 \mathrm{~s}$ and $\mathrm{T}=0.5 \mathrm{~s}$, and $5 \%$ for $\mathrm{T}=0.01 \mathrm{~s}$. The rise times $t_{r}$ are $0.91 \mathrm{~s}, 5.99 \mathrm{~s}$, and $8.86 \mathrm{~s}$, which mean that the designed discrete-time systems sample 90, 30, and 18 times per rise time, respectively.

The closed-loop step responses to a $1 \%$ step in the reference signal $h_{\text {ref }}$ for all designed controllers are shown in Fig. 13. The closed-loop control loops show overdamped or slightly underdamped responses with (dominant) time constants growing with $T$. Performance of the designed pressure control schemes with different bandwidths and sampling intervals was tested under time-varying conditions with a transient simulation model. For this purpose the authors generated separate realistic demand profiles for all nodes of the WDN which take into account random smaller intensity flow fluctuations from residential areas as well as larger sudden demands from an industrial and due to hydrant opening.

\section{Demand Profile}

The residential demand profile was generated for each demand node of the network using the bottom up stochastic approach by Buchberger and Wells (1996) where the demand events in a residence are represented by a series of rectangular pulses, each with an arrival time, an intensity, and a duration. As in Buchberger and Wells (1996) the arrival times were represented by a Poisson process. The duration and the intensity of each event were assigned random values according to exponential and normal distributions, respectively, as in Prescott and Ulanicki (2008). It is assumed that each of the 25 demand nodes, i.e. nodes 4 to 28 in Fig. 4, supplies 120 houses giving a total of 3,000 houses. A series of Poisson events was generated for each house over the duration time of 1000s. Parameter values from Prescott and Ulanicki (2008) were used, i.e. mean inter-event 
time of $5 \mathrm{~min}$, average event duration of $15 \mathrm{~s}$ and average event intensity of $6 \mathrm{~L} / \mathrm{min}$. The resulting demand pulse series for each house were then aggregated to yield a residential demand pattern for each node. In addition, two rapid water uptake events were added to the demand profiles in nodes 13 and 20. In node 20, a hydrant is opened after 100 seconds producing a 10L/s increase in water demand over a 5 s period and remaining at this value for 200 s, after which the hydrant is shut down, again over a 5 s period. A smaller and a more gradual increase in water demand is simulated in node 13 where a single industrial user is assumed to create a $5 \mathrm{~L} / \mathrm{s}$ increase in demand over 20 s at the simulation time of $500 \mathrm{~s}$, remaining at this value for further $200 \mathrm{~s}$ and decreasing to $0 \mathrm{~L} / \mathrm{s}$ over the next 20 seconds. The two above events were added to the residential profiles in nodes 13 and 20, respectively. The total (aggregated) demand profile is shown in Fig. 14

\section{Results}

The simulated pressure heads $h_{d}$ downstream of the valve for the selected closed-loop systems with sampling times $T=0.01 \mathrm{~s}, 0.5 \mathrm{~s}, 5 \mathrm{~s}$, and 60s are plotted in Fig. 15. As suspected, the higher the sampling time (and the lower the target closed-loop crossover frequency $\omega_{c}$ to guarantee the desired stability margins) the slower the response time of the system to incoming disturbances, i.e. pressure waves caused by demand changes. At $\mathrm{T}=0.5 \mathrm{~s}$, the controller is able to maintain $h_{d} \approx h_{\text {set }}=140 \mathrm{~m}$ throughout the simulation but temporary variations up to $\sim 20 \mathrm{~m}$ and lasting for about 20 s are visible as the system is unable to counteract the effects of incoming pressure waves. At $T=5 \mathrm{~s}$ the sudden variations are slightly higher and up to $\sim 30 \mathrm{~m}$ whilst at the same time the system takes longer to recover and settle to the setpoint. At $T=60$ s the pressure changes are up to $\sim 30-35 \mathrm{~m}$ but the closed-loop bandwidth is small enough that also lower frequency disturbances are not rejected and the periods with inadequate downstream pressure last for up to 200s. At longer sampling times in the range of minutes, it is expected that the periods with inadequate pressure will become longer depending on the frequency characteristics of the demand pattern in the network. Ultimately, at $T=0.01 \mathrm{~s}$ the controller designed with target $\omega_{c}=2 \mathrm{rad} / \mathrm{s}$ is able to better reject higher frequency disturbances and consequently, $h_{d}$ stays closer to the setpoint without longer term divergences and only occasional $\pm 4 \mathrm{~m}$ variations lasting for seconds. At even larger closed-loop bandwidths, it is 
expected that the disturbance rejection characteristics of the system will improve further and thus, the variability of the outlet pressure will be reduced even more. However, the overall performance will depend on the designed controller and its gains and the speed of the actuator which will need to be set following formal controller design procedures and testing. Variability in the output $y=h_{d}$ was measured as a mean integral squared error (MISE) defined as $e_{y, M I S E}=\frac{1}{t_{\text {simu }}} \int_{0}^{t_{\text {simu }}}\left(\frac{h_{d}(t)-h_{\text {ref }}}{h_{\text {ref }}}\right)^{2} d t$, where $t_{\text {simu }}$ is the simulation time and $h_{\text {ref }}$ is the reference signal (pressure setpoint). Control effort was measured as mean sum of squares (MSS): $Q_{u, M S S}=\frac{1}{N} \sum_{i=1}^{i=N}\left(\frac{x_{m}^{i}-x_{m, 0}}{x_{m, 0}}\right)^{2}$ and mean sum of absolute derivatives (MSAD): $Q_{u, M S A D}=\frac{1}{t_{\text {simu }}} \sum_{i=2}^{i=N}\left|\frac{x_{m}^{i}-x_{m}^{i-1}}{x_{m, 0}}\right|$, where $x_{m}^{i}$ denotes the valve position at the $i$-th time instant, $N$ is the number of discrete-time instants, and $x_{m, 0}$ is the (steady-state) valve position at the beginning of the simulation. $Q_{u, M S S}$ measures the energy spent on actuating the valve, i.e. control costs, whilst $Q_{u, M S A D}$ measures the variability of the controller output and can be used to measure the expected intensity of the wear of the mechanical components, i.e. the actuator and the control element. $e_{y, M I S E}, Q_{u, M S S}$ and $Q_{u, M S A D}$ are plotted in Fig. 16 for all seven controllers with sampling times of $0.01,0.20,0.50,1,5,15$, and 60 seconds, respectively. As the feedback control system becomes slower (i.e. has a smaller closed-loop bandwidth), the larger the output errors get, as the system is unable to reject the disturbances of higher frequencies. On the other hand, faster controllers maintain the setpoint more closely but produce more control action resulting in higher values of $Q_{u, M S S}$ and $Q_{u, M S A D} \cdot Q_{u, M S S}$ is a measure of energy spend on actuation. We can see that an increase in $Q_{u, M S S}$ is substantially smaller than the improvement in the overall system performance. $Q_{u, M S A D}$, on the other hand, is a measure of the speed of movement of the valve element and determines the power requirements of the actuator. Fig. 16 shows that the plots for $Q_{u, M S A D}$ and $e_{y, M I S E}$ are of a similar shape but have opposite gradients. Achieving better disturbance rejection requires more powerful actuators and comes at a cost of higher wear due to more action, especially during time periods with large variability in the demands. It is intuitive to think that in time-periods with less variability in the demands, the additional amount of control action required by faster controllers compared to the slower ones should be proportionally smaller as the faster controller operating at shorter sampling times will be acting on smaller deviations in 
the error signal, whilst controllers operating at longer sampling times will eventually let the error signal build in the system and may require larger error corrections.

\section{CONCLUSION}

As demonstrated in this paper, closed-loop feedback control systems can be destabilized by increasing gain and/or via delays. Such delays may come from the properties inherent of the controlled system, e.g. backlash in mechanical systems; communication delays in the control loops; sensors located away from the actuators or, in case of discrete-time systems; from sampling. In discrete-time control the practical rule of thumb states that the sampling period $T \leq t_{r} / 10$ where $t_{r}$ is the closed-loop rise time. Analogous criterion can be formulated in frequency domain, where the Nyquist frequency $\omega_{N}=\pi / T$ needs to be larger than $10 \omega_{c}-50 \omega_{c}, \omega_{c}$ being the desired bandwidth of the closed-loop system. In case of our I controller designed in the first half of this paper with target $\omega_{c}=0.15 \mathrm{rad} / \mathrm{s}$, the resulting closed-loop $t_{r}=8 \mathrm{~s}-15 \mathrm{~s}$. Therefore, the sampling time $T \lesssim 0.8$ s should theoretically ascertain good performance in the worst-case scenario. Step experiments with the transient model exhibited good transient response at $T=1 \mathrm{~s}$ while the output closely resembled the desired response in continuous-time at $T=0.25 \mathrm{~s}$. The loss of stability occurred at $T \approx 10$ s, i.e. $T \approx t_{r} . T=1$ s corresponds to $\omega_{N} \approx 2.1 \omega_{c}$ based on $\omega_{c}$ of the nominal system.

The speed of closed-loop response, measured by response time in time-domain or closed-loop bandwidth $\omega_{c}$ in frequency-domain, can be decreased by reducing the open-loop gain, as demonstrated initially on a closed-loop feedback system with open-loop inertial dynamics. Consequently, long sampling times can be used in feedback systems whilst still maintaining closed-loop stability provided that the open-loop static gain is sufficiently small - the case present in the bulk of the published works on RTC of WDNs - see e.g. Creaco et al. (2019). Nevertheless, as the gain is decreased, the effects of feedback are reduced and the closed-loop system becomes less effective at rejecting disturbances and following setpoints at lower frequencies up to the point where the system can no longer meet the desired performance criteria.

Simulations with the transient hydraulic WDN model with time-varying demand patterns 
demonstrated how the speed of the response of the closed-loop system, calculated for stability and robustness for each sampling period, affects the ability of the pressure control system to maintain the desired outlet pressure under transient conditions. In case of $T=0.01 \mathrm{~s}$ the closed-loop bandwidth of $2 \mathrm{rad} / \mathrm{s}$ is large enough that the system is able to react to the bulk of the frequencies describing the wave (transient) dynamics and thus, the impacts of the incoming pressure waves are substantially reduced. Consequently, the outlet pressure is kept near the desired value. As the sampling time is increased, requiring lower closed-loop bandwidths, i.e. controller speeds, the closed-loop system becomes less responsive to the disturbances, which manifests itself with larger downstream pressure variations around the setpoint and longer times with inadequate outlet pressure.

Identification of the transient WDN model coupled with the electrically actuated PRV under study revealed that the relationship between the valve position and the downstream head could be described with a family of $4^{\text {th }}$-order transfer functions having similar dynamics at all operating points and different static gains which depend strongly on valve position. For controller design purposes, this family of transfer functions could be approximated with a single (nominal) transfer function multiplied by the gain vs. valve position curve, as suggested in Janus and Ulanicki (2018). The feedback controller could therefore be designed on the nominal transfer function and implemented in the real system in combination with the gain compensator whose purpose is to adjust the gain of the controller depending on the valve position, such that the overall static gain of the open-loop system is approximately constant at all operating points.

\section{DATA AVAILABILITY}

The following data, models, or code generated or used during the study are available from the corresponding author by request.

- Simple first order sampled system in Simulink ${ }^{\circledR}$ used to demonstrate the mechanisms of instabilities in feedback systems.

- PRV/WDN system in Simulink ${ }^{\circledR}$ used for model identification and final simulation under 
time-varying demand.

- Family of identified transfer functions used for controller design.

- Code for generation of demand patterns as stochastic series of pulses.

\section{REFERENCES}

AbdelMeguid, H., Skworcow, P., and Ulanicki, B. (2011). "Mathematical modelling of a hydraulic controller for PRV flow modulation." Journal of Hydroinformatics, 13(3), 374-389.

Berardi, L., Simone, A., Laucelli, D. B., Ugarelli, R. M., and Giustolisi, O. (2018). "Relevance of hydraulic modelling in planning and operating real-time pressure control: case of Oppegård municipality." Journal of Hydroinformatics, 20(3), 535-550.

Buchberger, S. G. and Wells, G. J. (1996). "Intensity, Duration, and Frequency of Residential Water Demands." Journal of Water Resources Planning and Management, 122(1).

Campisano, A., Creaco, E., and Modica, C. (2010). "RTC of Valves for Leakage Reduction in Water Supply Networks.” Journal of Water Resources Planning and Management, 136(1), 138-141.

Campisano, A., Modica, C., Reitano, S., Ugarelli, R., and Bagherian, S. (2016). "Field-Oriented Methodology for Real-Time Pressure Control to Reduce Leakage in Water Distribution Networks." Journal of Water Resources Planning and Management, 142(12).

Campisano, A., Modica, C., and Vetrano, L. (2012). "Calibration of Proportional Controllers for the RTC of Pressures to Reduce Leakage in Water Distribution Networks." Journal of Water Resources Planning and Management, 138(4), 377-384.

Creaco, E. (2017). "Exploring Numerically the Benefits of Water Discharge Prediction for the Remote RTC of WDNs." Water, 9, 961.

Creaco, E., Campisano, A., Fontana, N., Marini, G., Page, P., and Walski, T. (2019). "Real time control of water distribution networks: A state-of-the-art review." Water Research, 161, 517 530.

Creaco, E., Campisano, A., Franchini, M., and Modica, C. (2017). "Unsteady Flow Modeling of Pressure Real-Time Control in Water Distribution Networks." Journal of Water Resources Planning and Management, 143(9). 
Creaco, E., Campisano, A., and Modica, C. (2018). “Testing behavior and effects of PRVs and RTC valves during hydrant activation scenarios." Urban Water Journal, 15(3), 218-226.

Creaco, E. and Franchini, M. (2013). “A new algorithm for real-time pressure control in water distribution networks.” Water Supply, 13(4), 875-882.

Fontana, N., Giugni, M., Glielmo, L., Marini, G., and Zollo, R. (2018). "Real-Time Control of Pressure for Leakage Reduction in Water Distribution Network: Field Experiments." Journal of Water Resources Planning and Management, 144(3), 04017096.

Galuppini, G., Creaco, E., Toffanin, C., and Magni, L. (2019). "Service pressure regulation in water distribution networks." Control Engineering Practice, 86, 70 - 84.

Galuppini, G., Magni, L., and Creaco, E. (2020). "Stability and robustness of real-time pressure control in water distribution systems.” Journal of Hydraulic Engineering, 146(4), 04020023.

Giustolisi, O., Ugarelli, R. M., Berardi, L., Laucelli, D. B., and Simone, A. (2017). "Strategies for the electric regulation of pressure control valves." Journal of Hydroinformatics, 19(5), 621-639.

Giustolisi, O. and Walski, T. M. (2012). "Demand Components in Water Distribution Network Analysis." Journal of Water Resources Planning and Management, 138(4).

Janus, T. and Ulanicki, B. (2017). "Hydraulic Modelling for Pressure Reducing Valve Controller Design Addressing Disturbance Rejection and Stability Properties.” Procedia Engineering, 186, 635 - 642 XVIII International Conference on Water Distribution Systems, WDSA2016.

Janus, T. and Ulanicki, B. (2018). "Improving Stability of Electronically Controlled PressureReducing Valves through Gain Compensation.” Journal of Hydraulic Engineering, 144(8).

Jung, B. B. S., Boulos, P. F., and Wood, D. J. (2009). "Effect of pressure-sensitive demand on surge analysis." American Water Works Association Journal.

L. Ljung, ed. (1999). System Identification (2Nd Ed.): Theory for the User. Prentice Hall PTR, Upper Saddle River, NJ, USA.

Ljung, L. (2009). "Experiments with Identification of Continuous Time Models." IFAC Proceedings Volumes, 42(10), 1175 - 1180 15th IFAC Symposium on System Identification.

Madoński, R., Nowicki, M., and Herman, P. (2014). “Application of active disturbance rejection 
controller to water supply system." Proceedings of the 33rd Chinese Control Conference, 44014405 (July).

Malppan, P. J. and Sumam, K. (2015). "Pipe burst risk assessment using transient analysis in surge 2000." Aquatic Procedia, 4, 747 - 754 INTERNATIONAL CONFERENCE ON WATER RESOURCES, COASTAL AND OCEAN ENGINEERING (ICWRCOE'15).

Ogata, K. (2010). Modern Control Engineering. Pearson, 5th edition.

Page, P. R., Abu-Mahfouz, A. M., and Mothetha, M. L. (2017a). "Pressure Management of Water Distribution Systems via the Remote Real-Time Control of Variable Speed Pumps.” Journal of Water Resources Planning and Management, 143(8), 04017045.

Page, P. R., Abu-Mahfouz, A. M., and Yoyo, S. (2017b). "Parameter-Less Remote Real-Time Control for the Adjustment of Pressure in Water Distribution Systems.” Journal of Water Resources Planning and Management, 143(9), 04017050.

Page, P. R. and Creaco, E. (2019). “Comparison of Flow-Dependent Controllers for Remote RealTime Pressure Control in a Water Distribution System with Stochastic Consumption.” Water, 11(3).

Page, P. R., Zulu, S., and Mothetha, M. L. (2018). "Remote real-time pressure control via a variable speed pump in a specific water distribution system." Journal of Water Supply: Research and Technology-Aqua, 68(1), 20-28.

Phillips, C. L. and Harbor, R. D. (2000). Feedback Control Systems (4th Ed.). Prentice-Hall, Inc., Upper Saddle River, NJ, USA.

Prescott, S. L. and Ulanicki, B. (2003). "Dynamic Modeling of Pressure Reducing Valves.” Journal of Hydraulic Engineering, 129(10), 804-812.

Prescott, S. L. and Ulanicki, B. (2008). "Improved Control of Pressure Reducing Valves in Water Distribution Networks.” Journal of Hydraulic Engineering, 134(1), 55-65.

Rezaei, H., Ryan, B., and Stoianov, I. (2015). "Pipe failure analysis and impact of dynamic hydraulic conditions in water supply networks." Procedia Engineering, 119, 253 - 262 Computing and Control for the Water Industry (CCWI2015) Sharing the best practice in water management. 
Sigurd Skogestad, C. G. (2018). "Should we forget the Smith Predictor?." IFAC-PapersOnLine, 51(4), 769 - 774 3rd IFAC Conference on Advances in Proportional-Integral-Derivative Control PID 2018.

S.Jones, R.Collins, and J.Boxall (2015). "Do transients contribute to turbidity failures of water distribution systems?.” BHR Group - 12th International Conference on Pressure Surges (November).

Strogatz, S. (2007). Nonlinear Dynamics And Chaos. Studies in nonlinearity. Sarat Book House.

Thornton, J. and Lambert, A. (2010). "Managing pressures to reduce new breaks.

van Zyl, J. E. and Cassa, A. M. (2014). "Modeling Elastically Deforming Leaks in Water Distribution Pipes." Journal of Hydraulic Engineering, 140(2).

Vande Vegte, J. (1986). Feedback Control Systems. Prentice-Hall international editions. PrenticeHall, <https://books.google.es/books?id=JnJFPgAACAAJ $>$.

Vicente, D. J., Garrote, L., Sánchez, R., and Santillán, D. (2016). "Pressure Management in Water Distribution Systems: Current Status, Proposals, and Future Trends.” Journal of Water Resources Planning and Management, 142(2), 04015061. 


\section{List of Tables}

1 Output $y_{k}$ and error $e_{k}$ values in the first 10 iterations of the step response of a closed-loop system given in Fig. 2 at two different sampling periods $T$ and openloop static gains $K \ldots \ldots \ldots \ldots$ 
TABLE 1. Output $y_{k}$ and error $e_{k}$ values in the first 10 iterations of the step response of a closed-loop system given in Fig. 2 at two different sampling periods $T$ and open-loop static gains K.

\begin{tabular}{cccccccc}
\hline \hline \multirow{2}{*}{ iter. $k$} & $r_{k}$ & \multicolumn{3}{c}{$K=2, T=4 \mathrm{~s}$} & \multicolumn{2}{c}{$K=1, T=4 \mathrm{~s}$} & \multicolumn{2}{c}{$K=2, T=\tau=0.4 \mathrm{~s}$} \\
& & $e_{k}$ & $y_{k}$ & $e_{k}$ & $y_{k}$ & $e_{k}$ & $y_{k}$ \\
\hline 0 & 0 & 0 & 0 & 0 & 0 & 0 & 0 \\
1 & 1 & 1 & 2 & 1 & 1 & 1 & 1.264 \\
2 & 1 & -1 & -2 & 0 & 0 & -0.264 & 0.131 \\
3 & 1 & 3 & 6 & 1 & 1 & 0.869 & 1.147 \\
4 & 1 & -5 & -10 & 0 & 0 & -0.147 & 0.236 \\
5 & 1 & 11 & 22 & 1 & 1 & 0.764 & 1.052 \\
6 & 1 & -21 & -42 & 0 & 0 & -0.052 & 0.321 \\
7 & 1 & 43 & 86 & 1 & 1 & 0.679 & 0.977 \\
8 & 1 & -85 & -170 & 0 & 0 & 0.023 & 0.389 \\
9 & 1 & 171 & 342 & 1 & 1 & 0.611 & 0.916 \\
\hline \hline
\end{tabular}




\section{List of Figures}

1 Control block diagram of a closed-loop pressure control scheme in a WDN using an actuated PRV coupled with an analogue electronic controller receiving: (a) continuous error signal $e(t)$ and (b) error signal after a sample \& hold block $\bar{e}(t) . \quad .40$

2 Feedback control system with first-order dynamics with time constant $\tau=0.4 \mathrm{~s}$, and open-loop static gain $K=2$ and a sample and $\mathrm{ZOH}$ block with sampling period

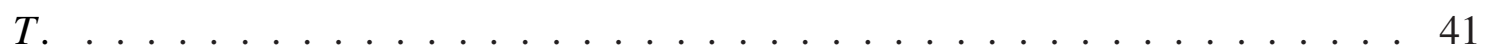

3 Time response $y$ of a closed-loop system given in Fig. 2 to a unit step input in $r$ at two different sampling periods $T \ldots \ldots \ldots$. . . . . . . . . . . 42

3a Time response at a sampling period $T=4$ s . . . . . . . . . 42

3b Time response at a sampling period $T=0.4 \mathrm{~s} \ldots \ldots \ldots$

4 Schematic diagram of the transient WDN simulation model's topology. . . . . . 43

5 Outlet pressure responses $\Delta h_{d}=h_{d}-h_{d, 0}$ from the transient model (bold) and the fitted transfer functions (thin) to a $1 \%$ step change in valve position $x_{m}$ at different pressure-dependent demand coefficients $C$ and pressure independent demands $d^{p i}$. 44 5a $\quad C=2 \times 10^{-6} \mathrm{~m}^{2} / \mathrm{s} \ldots \ldots \ldots \ldots \ldots \ldots$

5b $\quad C=8 \times 10^{-6} \mathrm{~m}^{2} / \mathrm{s} \ldots \ldots \ldots \ldots \ldots \ldots$

6 Frequency characteristics of the family of transfer functions representing the actuator and WDN dynamics at different operating points. . . . . . . . . . . . . 45

6a Original transfer functions. . . . . . . . . . . . . . 45

$6 \mathrm{~b} \quad$ Gain compensated transfer functions. . . . . . . . . . . . . 45

7 Dependency of the gain $K$ in the family of identified transfer functions representing the actuator and the WDN system at different operating points on valve position $x_{m} . \quad 46$

8 Pole locations in the $\mathcal{Z}$-plane for the nominal discrete-time closed-loop system $H_{\text {closed }}(z)$ at different sampling periods $T \ldots \ldots$. . . . . . . . . 47 
9 Closed loop responses of the sampled system with different sampling periods $T$ and at different operating points represented by different identified transfer function

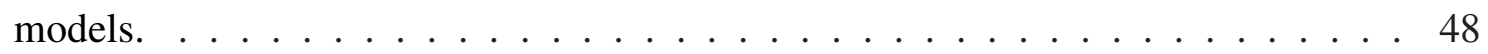

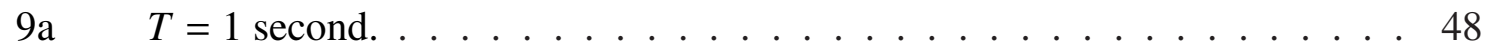

$9 \mathrm{~b} \quad T=5$ seconds. . . . . . . . . . . . . . . . 48

9 c $\quad T=10$ seconds. . . . . . . . . . . . . . . 48

$9 \mathrm{~d} \quad T=15$ seconds. . . . . . . . . . . . . . . 48

10 Closed loop responses of the sampled system with different sampling periods $T$ and at different operating points using the transient simulation model. . . . . . . . 49

10a $\quad T=1$ second. . . . . . . . . . . . . . . 49

10b $\quad T=5$ seconds. . . . . . . . . . . . . . . 49

10c $\quad T=10$ seconds. . . . . . . . . . . . . . . 49

10d $T=0.25$ seconds. . . . . . . . . . . . . . . 49

11 Bode plots of the open-loop system used to generate closed-loop step responses showing dependence of phase margins (PM) and gain margins (GM) on sampling time $T$ and static gain $K \ldots \ldots \ldots \ldots \ldots \ldots$

11a Magnitude and phase characteristics at different sampling times $T$. . . . . 50

11b Magnitude and phase characteristics at different static gains $K \ldots \ldots$. . . 50

12 Phase and gain margins for systems designed with different target sampling times $T$ and different target closed-loop bandwidths $\omega_{c}$ defined by a fraction $f_{\omega_{c}}$ of Nyquist frequency $\pi / T \ldots \ldots \ldots \ldots \ldots \ldots$

13 Closed loop responses of the sampled systems with different controllers and different sampling periods $T \ldots \ldots \ldots \ldots \ldots \ldots \ldots$

14 Total pressure-independent demand profile across the WDN used in the case study. 53

15 Downstream head $h_{d}$ during simulation under time-varying demands in a closedloop pressure control system with different controllers and sampling times. . . . . . 54 
16 Errors in the output signal $y=h_{d}$ (measured as mean integral squared error (MISE), and control effort $u=x_{m}$, measured as mean sum of squares (MSS) and mean sum of absolute derivatives (MSAD) for feedback control systems implemented with different controllers and with different sampling periods $T$. . . . . . . . . . 55 


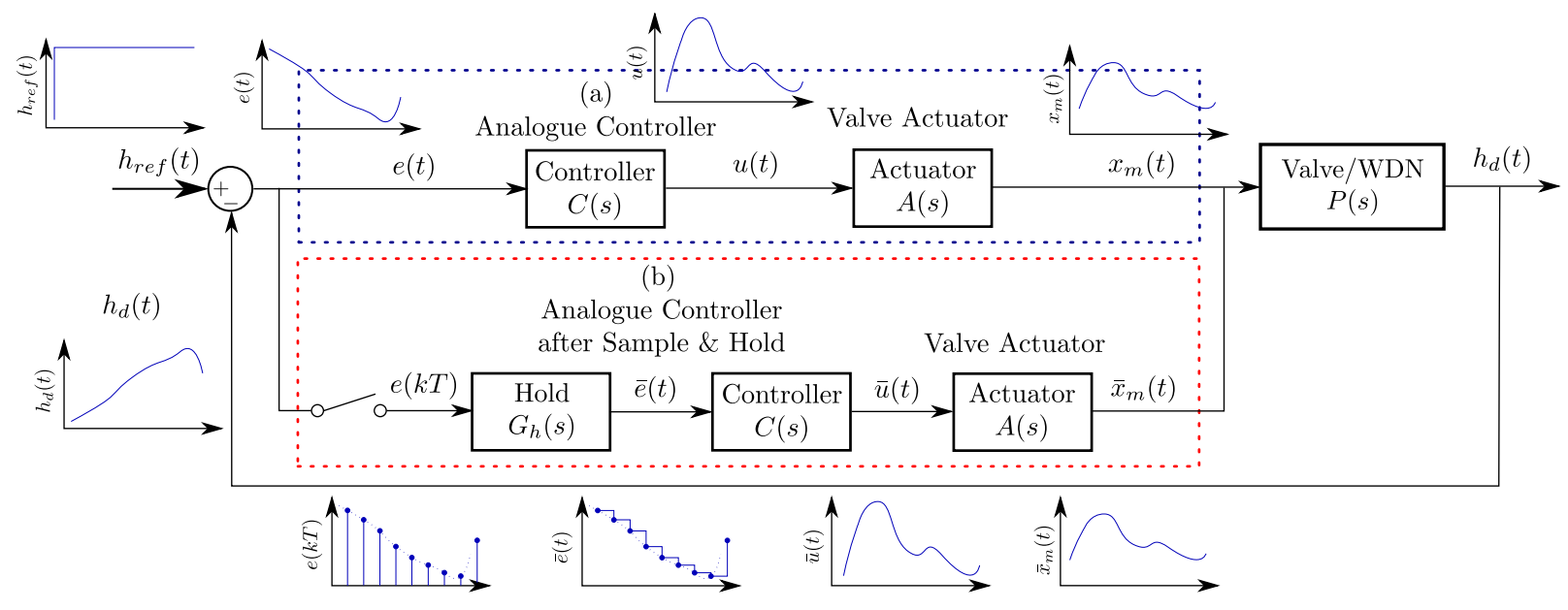

Fig. 1. Control block diagram of a closed-loop pressure control scheme in a WDN using an actuated PRV coupled with an analogue electronic controller receiving: (a) continuous error signal $e(t)$ and (b) error signal after a sample $\&$ hold block $\bar{e}(t)$. 


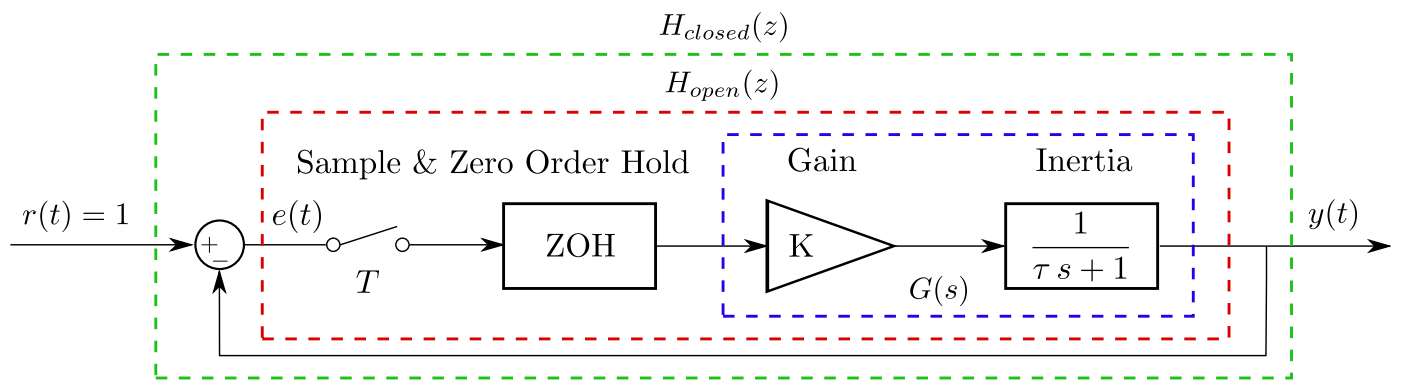

Fig. 2. Feedback control system with first-order dynamics with time constant $\tau=0.4 \mathrm{~s}$, and open-loop static gain $K=2$ and a sample and $\mathrm{ZOH}$ block with sampling period $T$. 


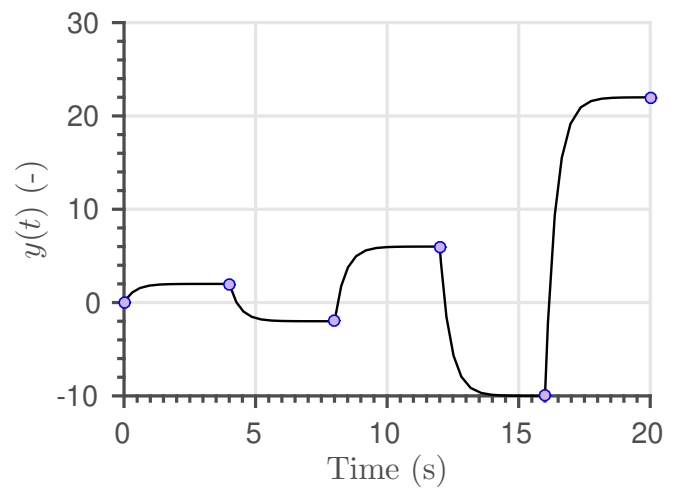

(a) Time response at a sampling period $T=4 \mathrm{~s}$

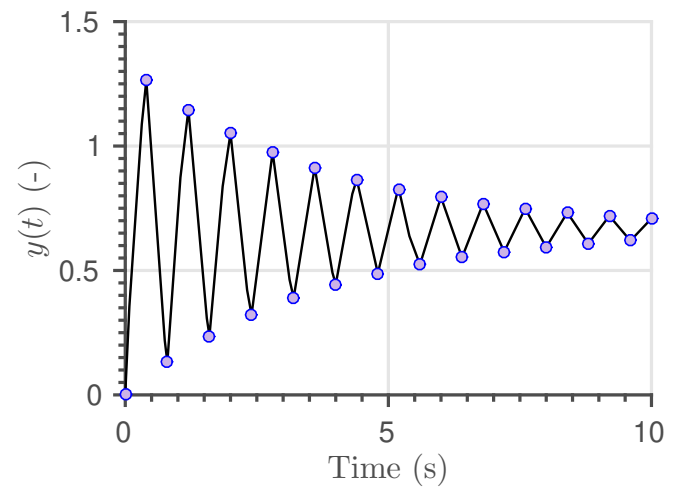

(b) Time response at a sampling period $T=0.4 \mathrm{~s}$

Fig. 3. Time response $y$ of a closed-loop system given in Fig. 2 to a unit step input in $r$ at two different sampling periods $T$. 


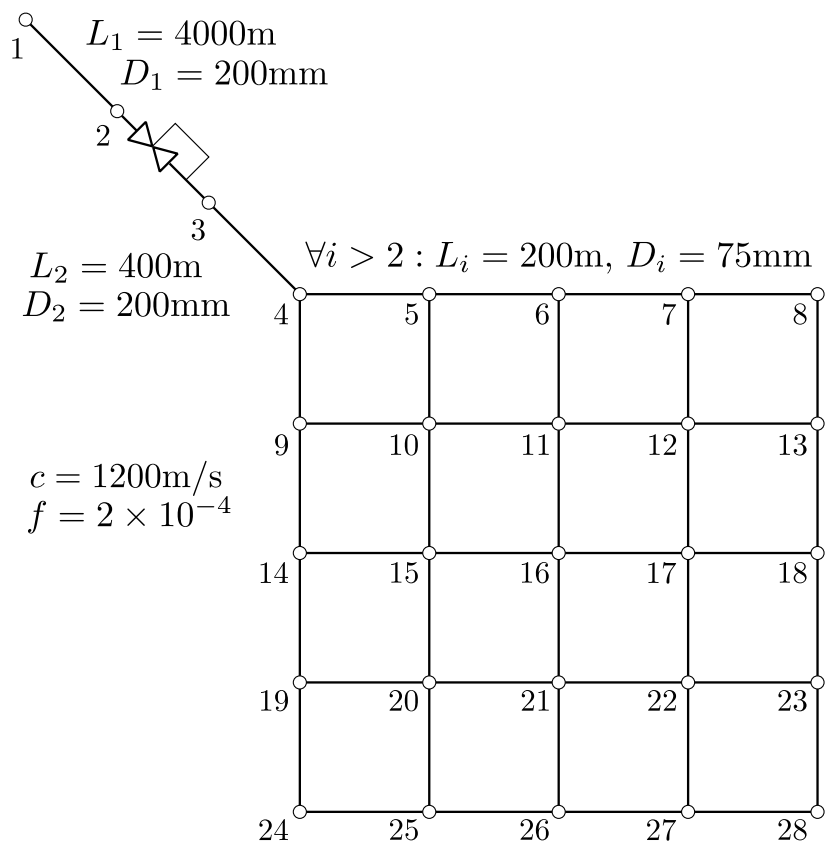

Fig. 4. Schematic diagram of the transient WDN simulation model's topology. 


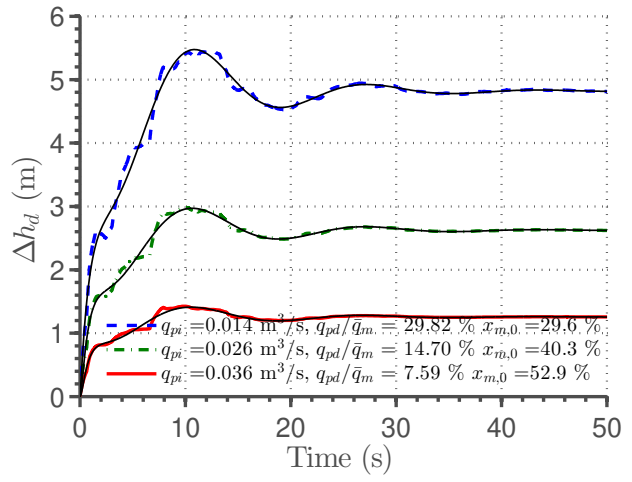

(a) $C=2 \times 10^{-6} \mathrm{~m}^{2} / \mathrm{s}$

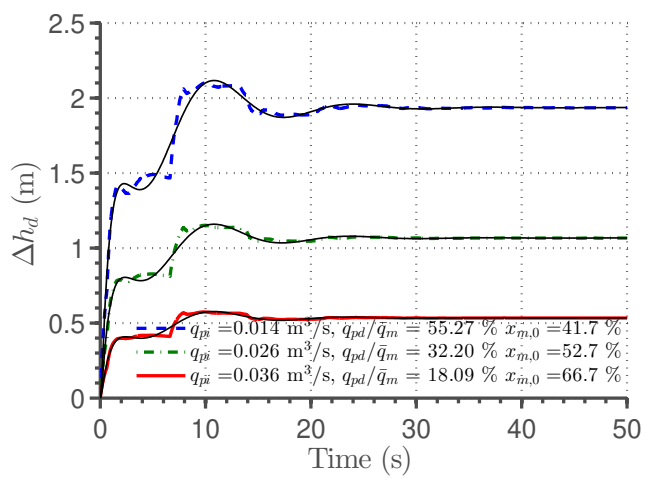

(b) $C=8 \times 10^{-6} \mathrm{~m}^{2} / \mathrm{s}$

Fig. 5. Outlet pressure responses $\Delta h_{d}=h_{d}-h_{d, 0}$ from the transient model (bold) and the fitted transfer functions (thin) to a $1 \%$ step change in valve position $x_{m}$ at different pressure-dependent demand coefficients $C$ and pressure independent demands $d^{p i}$. 


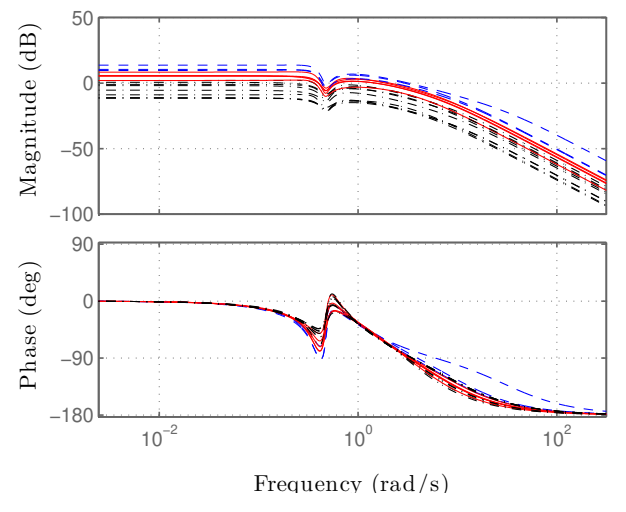

(a) Original transfer functions.

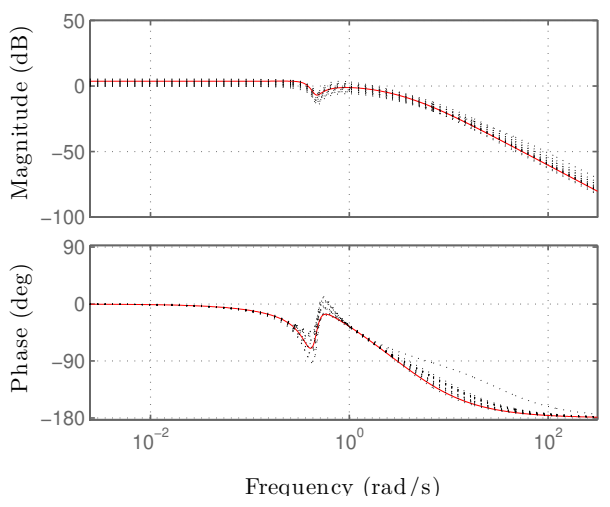

(b) Gain compensated transfer functions.

Fig. 6. Frequency characteristics of the family of transfer functions representing the actuator and WDN dynamics at different operating points. 


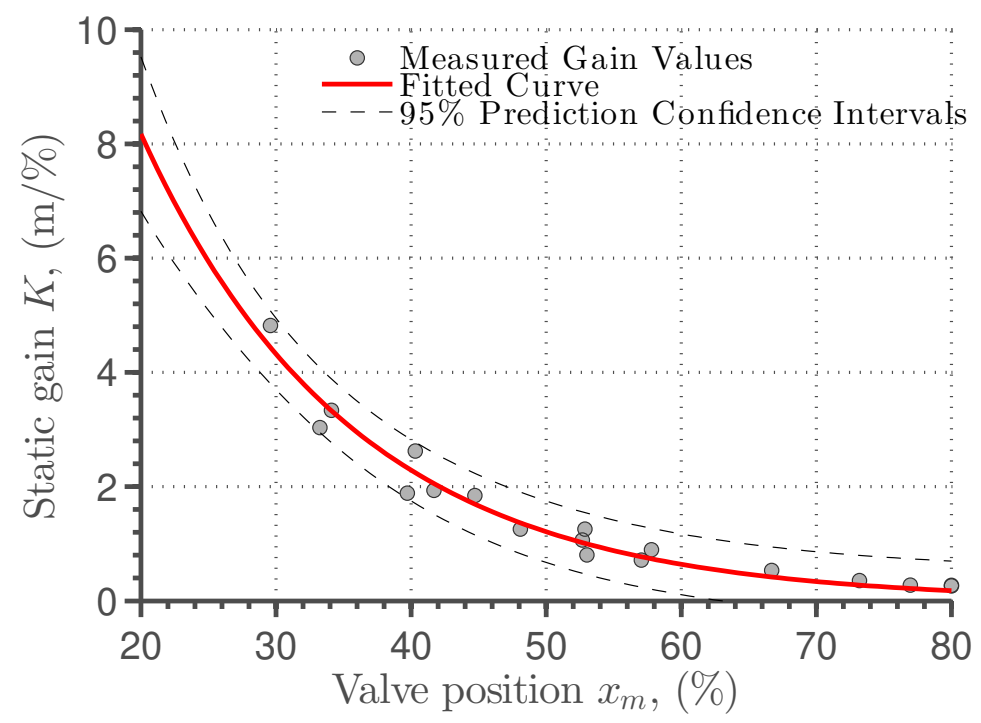

Fig. 7. Dependency of the gain $K$ in the family of identified transfer functions representing the actuator and the WDN system at different operating points on valve position $x_{m}$. 


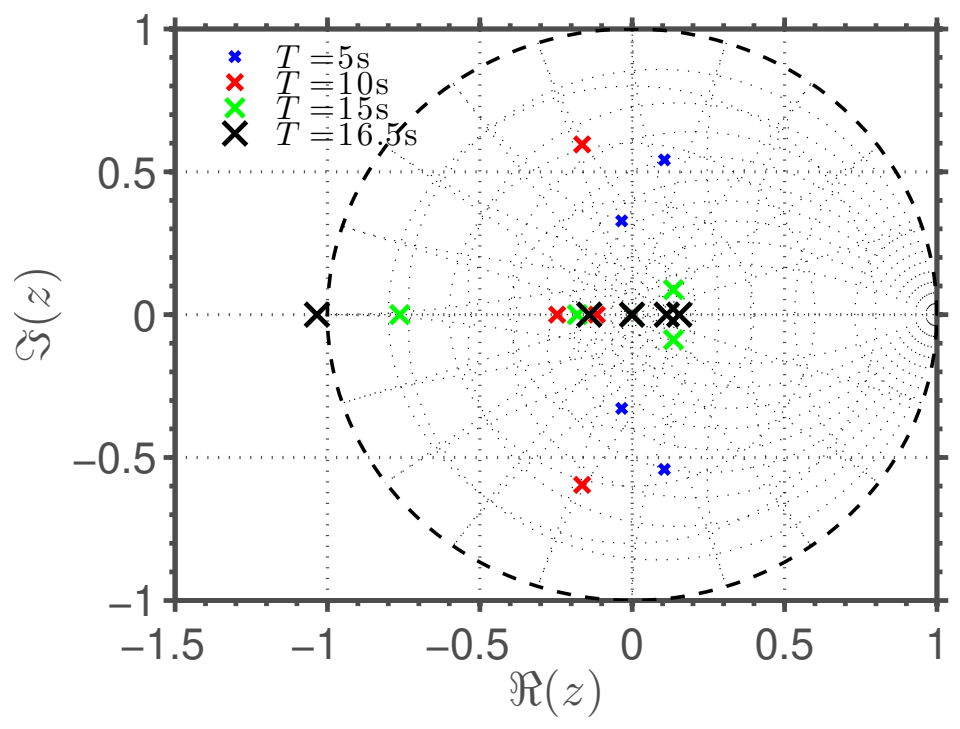

Fig. 8. Pole locations in the $\mathcal{Z}$-plane for the nominal discrete-time closed-loop system $H_{\text {closed }}(z)$ at different sampling periods $T$ 


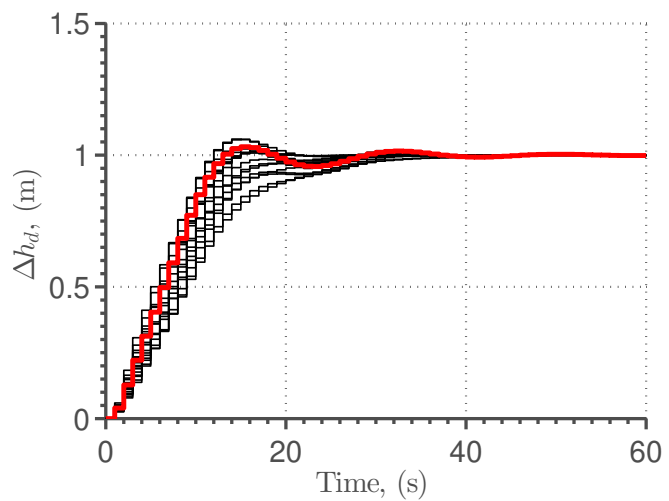

(a) $T=1$ second.

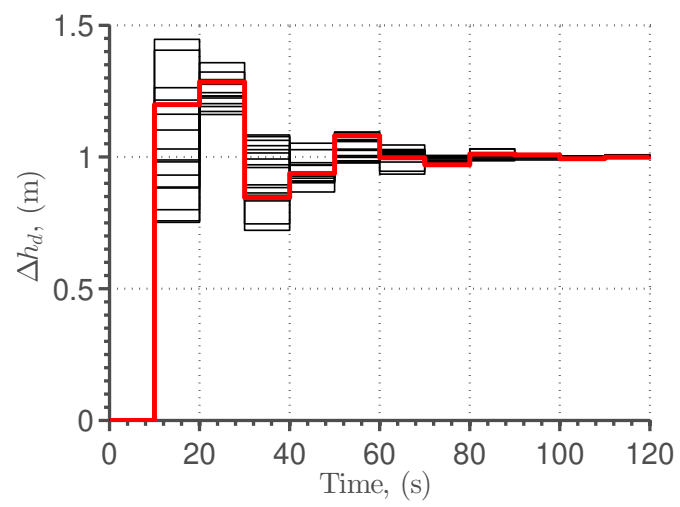

(c) $T=10$ seconds.

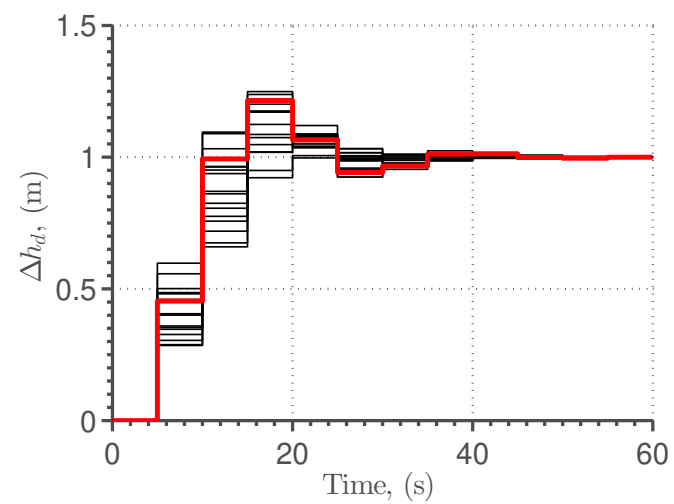

(b) $T=5$ seconds.

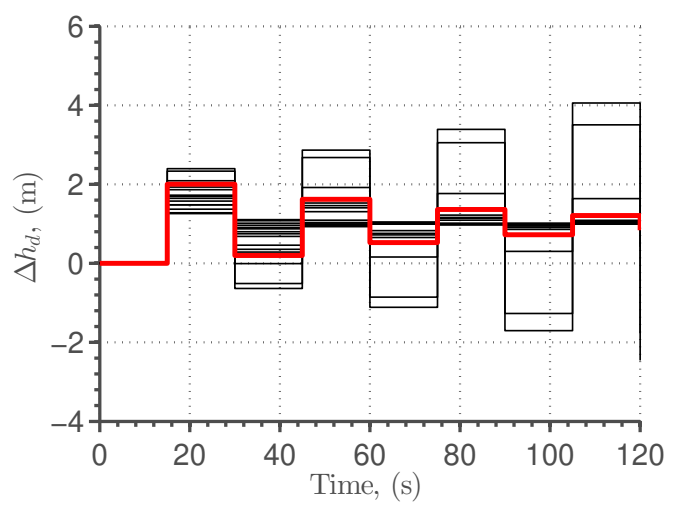

(d) $T=15$ seconds.

Fig. 9. Closed loop responses of the sampled system with different sampling periods $T$ and at different operating points represented by different identified transfer function models. 


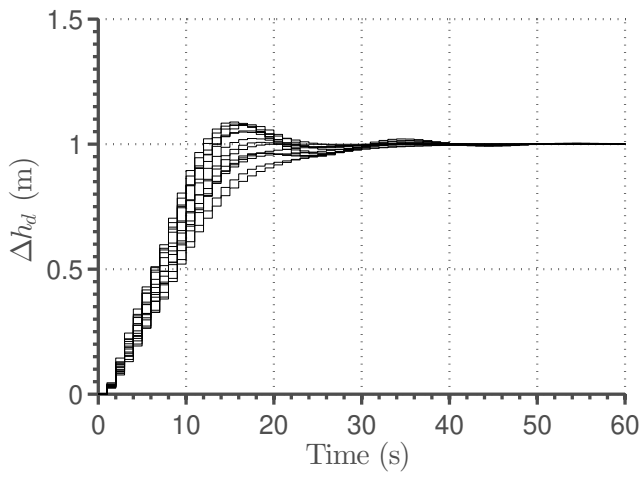

(a) $T=1$ second.

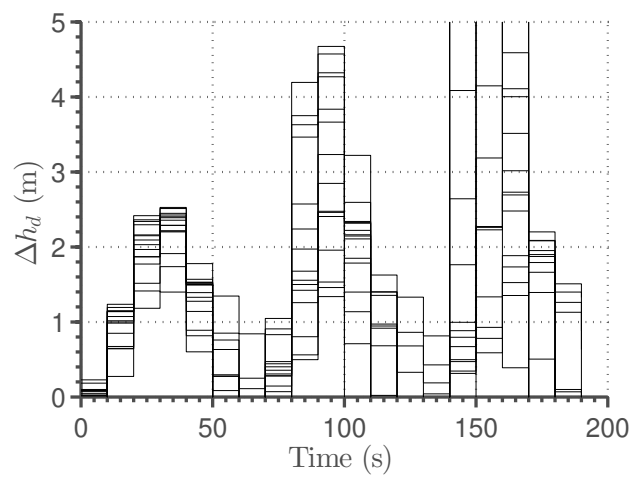

(c) $T=10$ seconds.

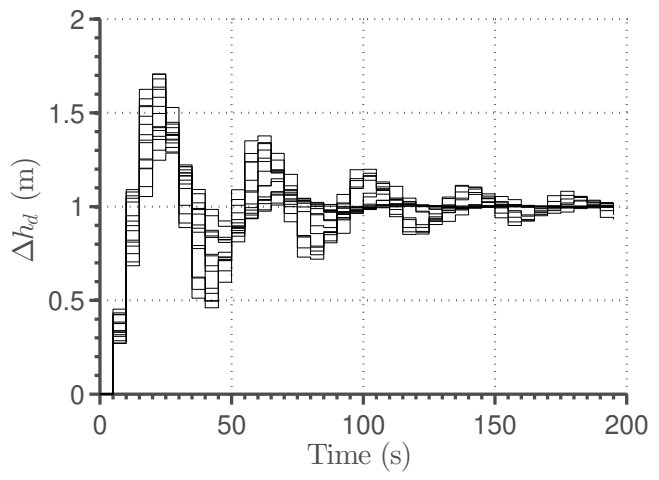

(b) $T=5$ seconds.

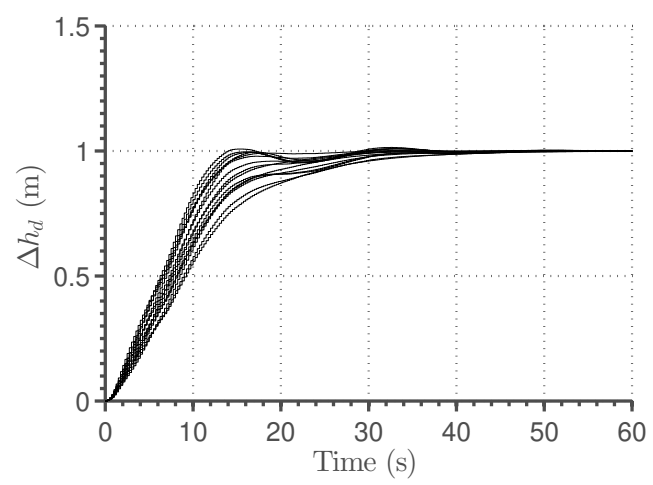

(d) $T=0.25$ seconds.

Fig. 10. Closed loop responses of the sampled system with different sampling periods $T$ and at different operating points using the transient simulation model. 

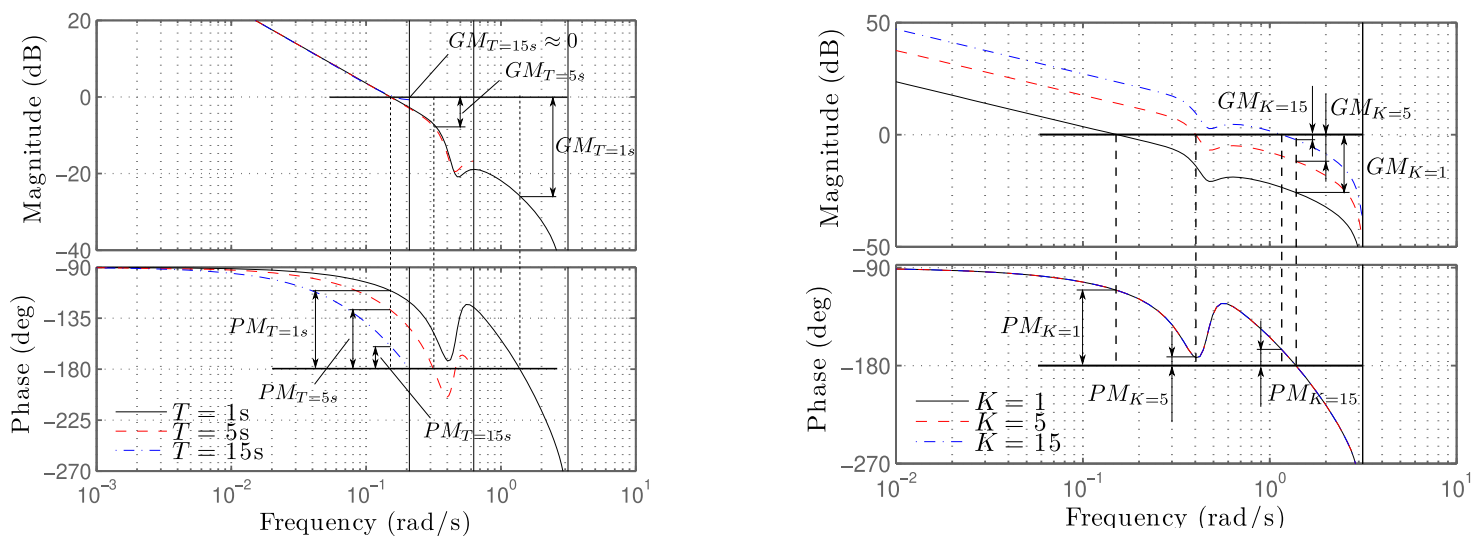

(a) Magnitude and phase characteristics at different (b) Magnitude and phase characteristics at different sampling times $T$. static gains $K$.

Fig. 11. Bode plots of the open-loop system used to generate closed-loop step responses showing dependence of phase margins (PM) and gain margins (GM) on sampling time $T$ and static gain $K$ 

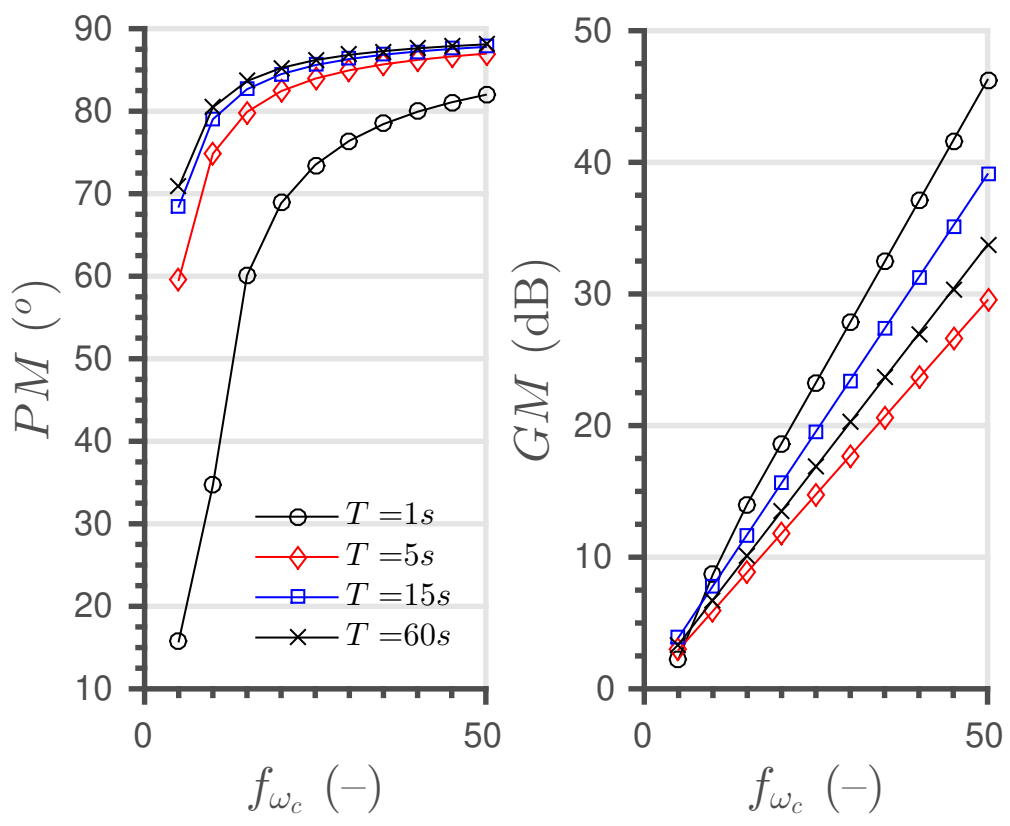

Fig. 12. Phase and gain margins for systems designed with different target sampling times $T$ and different target closed-loop bandwidths $\omega_{c}$ defined by a fraction $f_{\omega_{c}}$ of Nyquist frequency $\pi / T$. 

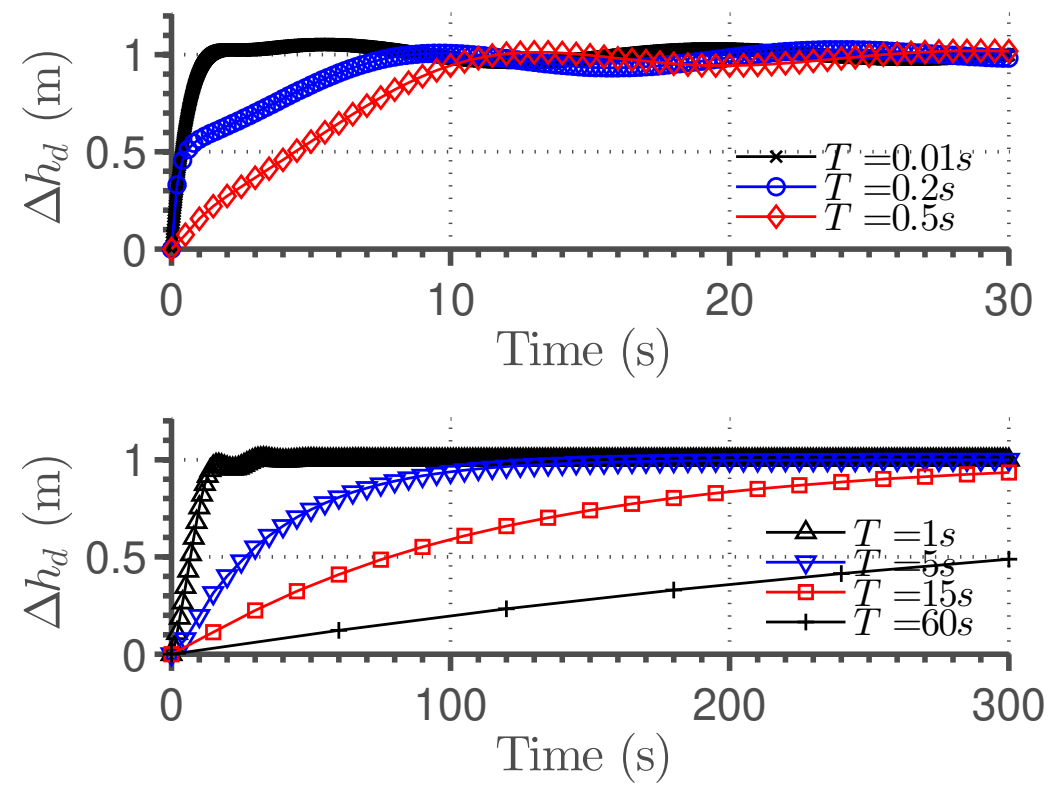

Fig. 13. Closed loop responses of the sampled systems with different controllers and different sampling periods $T$. 


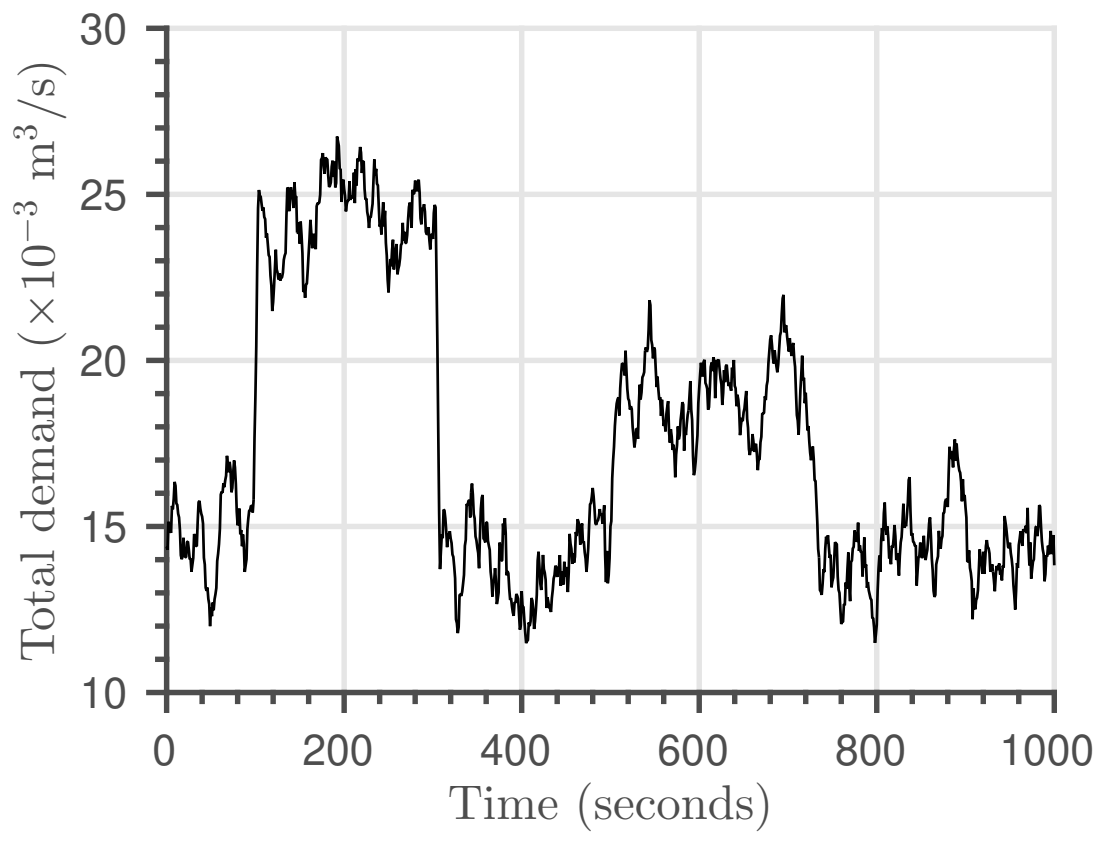

Fig. 14. Total pressure-independent demand profile across the WDN used in the case study. 

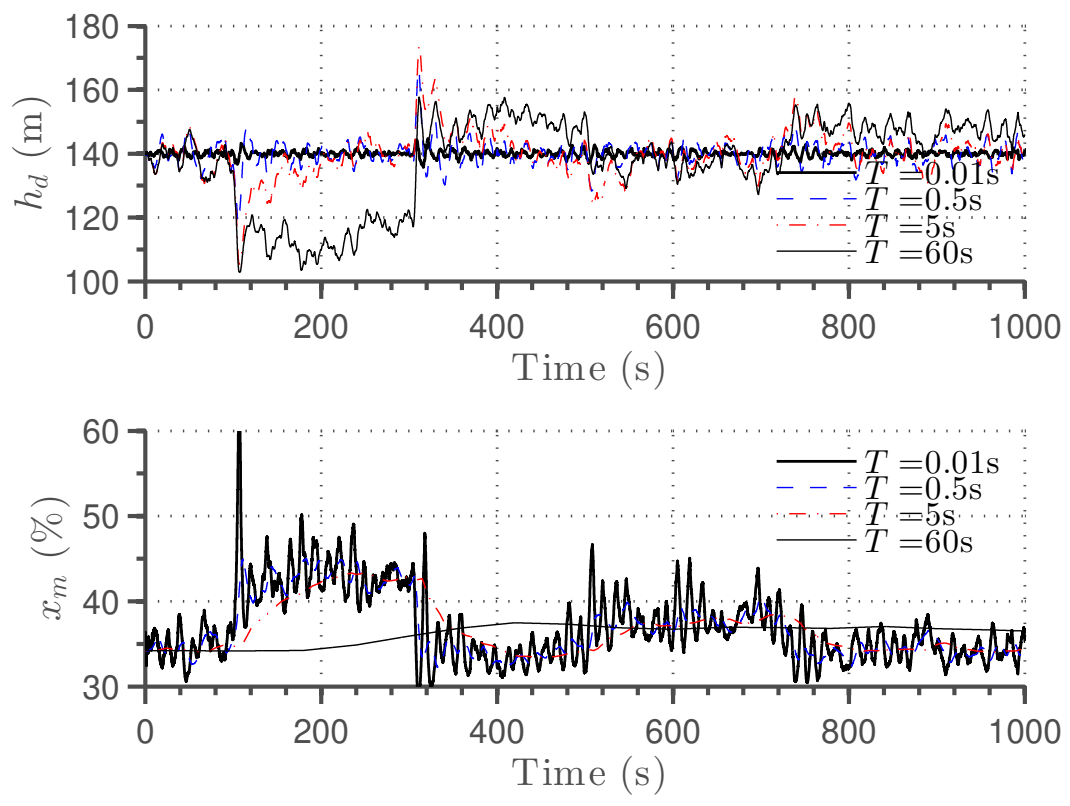

Fig. 15. Downstream head $h_{d}$ during simulation under time-varying demands in a closed-loop pressure control system with different controllers and sampling times. 


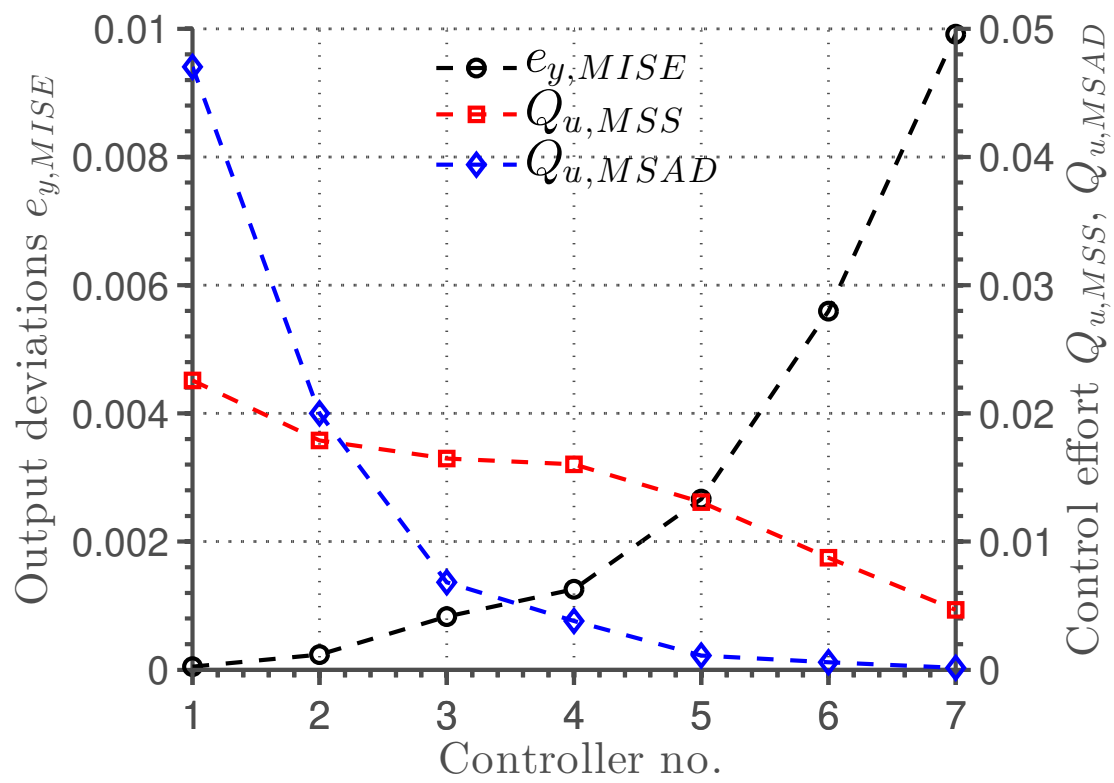

Fig. 16. Errors in the output signal $y=h_{d}$ (measured as mean integral squared error (MISE), and control effort $u=x_{m}$, measured as mean sum of squares (MSS) and mean sum of absolute derivatives (MSAD) for feedback control systems implemented with different controllers and with different sampling periods $T$. 